\title{
SPITZER SPACE TELESCOPE OBSERVATIONS OF MAGNETIC CATACLYSMIC VARIABLES: POSSIBILITIES FOR THE PRESENCE OF DUST IN POLARS
}

\author{
C. S. Brinkworth, D. W. Hoard, and S. Wachter \\ Spitzer Science Center, California Institute of Technology, Pasadena, CA 91125 \\ S. B. Howell \\ WIYN Observatory/National Optical Astronomy Observatory, Tucson, AZ 85719 \\ DAVID R. CIARDI \\ Michelson Science Center, California Institute of Technology, Pasadena, CA 91125 \\ P. SZKODY \\ Department of Astronomy, University of Washington, Seattle, WA 98195-1580 \\ T. E. HARRISON \\ Department of Astronomy, New Mexico State University, Las Cruces, NM 88003 \\ G. T. van Belle \\ Michelson Science Center, California Institute of Technology, Pasadena, CA 91125 \\ AND \\ A. A. EsIN \\ Department of Physics, Harvey Mudd College, Claremont, CA 91711-5990 \\ Received 2006 August 8; accepted 2007 January 10
}

\begin{abstract}
We present Spitzer photometry of six short-period polars, EF Eri, V347 Pav, VV Pup, V834 Cen, GG Leo, and MR Ser. We have combined the Spitzer IRAC $(3.6-8.0 \mu \mathrm{m})$ data with the 2MASS $J H K_{s}$ photometry to construct the SEDs of these systems from the near- to mid-IR $(1.235-8 \mu \mathrm{m})$. We find that five out of the six polars have flux densities in the mid-IR that are substantially in excess of the values expected from the stellar components alone. We have modeled the observed SEDs with a combination of contributions from the white dwarf, secondary star, and either cyclotron emission or a cool, circumbinary dust disk to fill in the long-wavelength excess. We find that a circumbinary dust disk is the most likely cause of the $8 \mu \mathrm{m}$ excess in all cases, but we have been unable to rule out the specific (but unlikely) case of completely optically thin cyclotron emission as the source of the observed $8 \mu \mathrm{m}$ flux density. While both model components can generate enough flux at $8 \mu \mathrm{m}$, neither dust nor cyclotron emission alone can match the excess above the stellar components at all wavelengths. A model combining both cyclotron and dust contributions, possibly with some accretion-generated flux in the near-IR, is probably required, but our observed SEDs are not sufficiently well sampled to constrain such a complicated model. If the $8 \mu \mathrm{m}$ flux density is caused by the presence of a circumbinary dust disk, then our estimates of the masses of these disks are many orders of magnitude below the mass required to affect $\mathrm{CV}$ evolution.
\end{abstract}

Subject headings: infrared: stars — stars: low-mass, brown dwarfs — stars: magnetic fields

\section{INTRODUCTION}

Cataclysmic variables (CVs) are semidetached binary systems consisting of a white dwarf (WD) primary star that accretes material from a low-mass secondary star, with typical orbital periods of $P_{\text {orb }} \lesssim 1$ day. Due to its large specific orbital angular momentum, the mass lost from the secondary star does not fall directly onto the WD. In most CVs, it instead settles into a disk around the primary star before losing enough angular momentum through viscous processes to finally accrete onto the WD. The release of gravitational potential energy in the disk causes it to be, by far, the brightest component of a CV over a wide range of wavelengths. On the other hand, polars (or AM Her stars, after the prototype) are CVs containing a WD with a strong $(B \geq 10 \mathrm{MG})$ magnetic field. The matter lost from the secondary star is captured by the field lines and funneled onto the magnetic pole(s) of the WD. The infalling matter is shocked as it nears the surface of the WD, radiating over most of the electromagnetic spectrum from X-rays to infrared. Observational characteristics and types of CVs are extensively reviewed by Warner (1995).
Polars are highly variable, both at a low level (usually $\sim 1-2$ mag) over their orbital periods as the geometry of the system changes along the line of sight and on much longer timescales with much greater changes in luminosity (up to $\sim 4 \mathrm{mag}$ ). Until recently, polars were generally thought to hover around a maximum brightness, occasionally switching into relatively short low states, with no preferred minimum brightness for a given system. The cause of these low states is not fully understood but is possibly due to the presence of star spots on the surface of the secondary star passing across the L1 point and suppressing mass transfer into the Roche lobe of the WD (Hessman et al. 2000). The resultant lowered accretion rate leads to a period of lower luminosity until the star spot has moved away from the L1 point and normal mass transfer resumes. More recently, however, a number of polars have been seen to hover in low states (Gerke \& Howell 2006; Araujo-Betancor et al. 2005), suggesting that the true cause of the accretion state changes in these CVs is more complex than previously believed.

The secular evolution of CVs is driven by the loss of orbital angular momentum, which causes the separation of the two 
component stars (and, hence, their orbital period) to continually decrease. While the secondary star radius decreases with mass loss, this simultaneous draining of angular momentum from the system keeps it in contact with its Roche lobe and enables the ongoing transfer of matter to the WD. Observationally, this results in CVs with shorter orbital periods having secondary stars with correspondingly later spectral types (i.e., lower masses). Eventually this ongoing mass loss from the secondary star causes it to drop below the hydrogen-burning limit, and the secondary star becomes a degenerate brown dwarf-like object. This change in the internal structure of the secondary star causes its radius to increase in response to further mass loss. This, in turn, results in a reversal of the trend of orbital period change, leading to a gradual increase in both $P_{\text {orb }}$ and binary separation. This is believed to be responsible for the observed minimum orbital period of CVs at $P_{\text {orb }} \sim 80$ minutes (Paczynski \& Sienkiewicz 1981; Rappaport et al. 1982; Howell et al. 2001).

Population synthesis studies predict that the secondary stars in $\sim 70 \%$ of all CVs should have evolved past the hydrogen-burning limit (e.g., Kolb 1993; Howell et al. 1997). Littlefair et al. (2003) reviewed all relevant observational data and analyses in the literature and concluded that while there is plausible indirect evidence for the existence of brown dwarf secondary stars in 39 systems, none of these had a reliable secondary star mass estimate or accurate enough spectral type to be certain. More importantly, they concluded that even if all of the potential candidates do indeed contain brown dwarf secondary stars, this only amounts to about $10 \%$ of all CVs, a huge deficit compared to the predicted $70 \%$ of $\mathrm{CVs}$. Consequently, it has become very important to ascertain the number of systems, if any, containing brown dwarf secondary stars, in order to place limits on our theories of $\mathrm{CV}$ population age and evolution.

Here we present Spitzer Space Telescope photometry of six polars that were identified as having very short orbital periods ( $P_{\text {orb }} \lesssim 90$ minutes) and/or very red Two Micron All Sky Survey (2MASS) colors (Hoard et al. 2002). This, along with other published evidence (e.g., compiled by Littlefair et al. 2003), suggests that they are good candidates for containing brown dwarf secondary stars. Our Spitzer observations were intended to enable us to unambiguously classify the spectral type of the secondary star by isolating its contribution to the mid-IR flux. However, as shown in Howell et al. (2006c, hereafter Paper I), even in the mid-IR the signature of the low-mass secondary star in these polars is contaminated by excess emission beyond the level expected from the stellar components. In this paper we explore in detail the possible origins of the mid-IR emission in magnetic CVs.

Preliminary results for four of our targets (EF Eridani, GG Leonis, V347 Pavonis, and RX J0154.0-5947) were presented in Paper I. The first three are presented again here with more extensive modeling for completeness of our entire sample of polars, but RX J0154.0-5947 is poorly studied and too little is known about its system parameters to warrant the more detailed modeling in this paper (in addition, it is the faintest of our polars; see Paper I). In addition, we present the first results from Spitzer mid-IR observations of the polars V834 Centauri, VV Puppis, and MR Serpentis.

\section{OBSERVATIONS AND DATA REDUCTION}

All of our mid-IR observations were obtained with the Infrared Array Camera (IRAC; Fazio et al. 2004) on the Spitzer Space Telescope (Werner et al. 2004). A log of the observations can be found in Table 1. The images were reduced and fluxcalibrated with the S12 version of the Spitzer IRAC pipeline and
TABLE 1

Log of Observations with the Spitzer Space Telescope

\begin{tabular}{cccc}
\hline \hline Polar & AOR Key & $\begin{array}{c}\text { Observation Date } \\
\text { (UT) }\end{array}$ & $\begin{array}{c}\text { Total Exposure Time } \\
(\mathrm{s})\end{array}$ \\
\hline V834 Cen...... & 10191360 & 2005 Jul 16 & 20 \\
EF Eri.......... & 10185984 & 2005 Jan 17 & 300 \\
GG Leo ........ & 13532160 & 2005 May 06 & 120 \\
V347 Pav ...... & 13531648 & 2005 Aug 19 & 300 \\
VV Pup ......... & 10191872 & 2004 Nov 26 & 120 \\
MR Ser......... & 10188288 & 2005 Mar 27 & 20 \\
\hline
\end{tabular}

downloaded from the archive as basic calibrated data (BCD). We obtained five BCD frames per object per wavelength channel, dithered with the medium-scale Gaussian pattern. The IRAC channels have central wavelengths of 3.6, 4.5, 5.8, and $8.0 \mu \mathrm{m}$ for channels $1-4$, respectively. The BCD images were corrected for array location dependency using the correction frames provided by the Spitzer Science Center. The frames were then combined with dual-outlier rejection to produce a mosaicked image for each channel using the Spitzer Science Center mosaicking software, MOPEX. ${ }^{1}$

We then performed IRAF ${ }^{2}$ aperture photometry on the mosaicked fields using an aperture size of 2.5 pixels. These results were subsequently corrected to an aperture size of 10 pixels using aperture corrections derived from 46 isolated sources in our fields. The values of the aperture correction factors are 1.190, $1.199,1.211$, and 1.295 for channels $1-4$, respectively. From experience we found that the point response function (PRF) for the IRAC arrays is not well modeled, and we obtained more consistent results using aperture photometry than via PRF fitting. Sky subtraction was tested in three different ways: (1) using a sky annulus around the target, (2) using a sky region a short distance from the target (only for the more crowded fields of EF Eri and VV Pup), and (3) subtracting a median sky from the whole mosaic before performing the photometry. The three methods were found to be consistent with each other. We extended the wavelength coverage for our data set into the near-IR using archival data from the 2MASS All Sky Point Source Catalogue (Skrutskie et al. 2006). The $2 \mathrm{MASS} J, H$, and $K_{s}$ magnitudes were converted to flux density in mJy using the absolute calibration zero points given in Cohen et al. (2003). All of the target photometry can be found in Table 2, and the resultant spectral energy distributions (SEDs) are shown in Figure 1.

\subsection{Error Analysis}

The only published attempts at rigorous error analysis for IRAC photometry are by Lacy et al. (2005), who derived absolute errors of $7 \%, 12 \%, 11 \%$, and $12 \%$ for channels $1-4$, respectively, and by Reach et al. (2005), who carried out an absolute calibration of the IRAC instrument. The analysis by Lacy et al. (2005) was calibrated relative to corresponding 2MASS flux densities in an effort to obtain an absolute uncertainty for the IRAC colors. Those results therefore incorporate the uncertainties in both the 2MASS photometry and the IRAC colors for their objects. As the absolute calibration for IRAC is likely to change as more data are obtained, we performed our own error analysis to

\footnotetext{
1 Mosaicking and Point-Source Extraction; see Makovoz \& Marleau (2005) and the IRAC Data Handbook for a comprehensive discussion of the MOPEX package and the dual-outlier rejection algorithm.

2 The Image Reduction and Analysis Facility is maintained and distributed by the National Optical Astronomy Observatory.
} 
TABLE 2

Measured 2MASS and IRAC Flux Densities (mJy)

\begin{tabular}{|c|c|c|c|c|c|c|c|}
\hline Polar & $\begin{array}{c}J \\
(1.2 \mu \mathrm{m})\end{array}$ & $\begin{array}{c}H \\
(1.7 \mu \mathrm{m})\end{array}$ & $\begin{array}{c}K_{s} \\
(2.2 \mu \mathrm{m})\end{array}$ & $\begin{array}{l}\text { IRAC-1 } \\
(3.6 \mu \mathrm{m})\end{array}$ & $\begin{array}{l}\text { IRAC-2 } \\
(4.5 \mu \mathrm{m})\end{array}$ & $\begin{array}{l}\text { IRAC-3 } \\
(5.8 \mu \mathrm{m})\end{array}$ & $\begin{array}{l}\text { IRAC-4 } \\
(8.0 \mu \mathrm{m})\end{array}$ \\
\hline V834 Cen ...................... & $6.54_{-0.16}^{+0.16}$ & $5.60_{-0.11}^{+0.11}$ & $5.22_{-0.12}^{+0.13}$ & $2.39_{-0.05}^{+0.05}$ & $2.33_{-0.06}^{+0.06}$ & $2.04_{-0.16}^{+0.16}$ & $2.48_{-0.15}^{+0.15}$ \\
\hline EF Eri............................ & $0.21_{-0.04}^{+0.100}$ & $0.55_{-0.08}^{+0.11}$ & $0.47_{-0.08}^{+0.08}$ & $0.71_{-0.02}^{+0.03}$ & $0.72_{-0.02}^{+0.00}$ & $0.66_{-0.07}^{+0.07}$ & $0.70_{-0.05}^{+0.13}$ \\
\hline 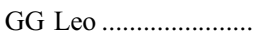 & $1.57_{-0.06}^{+0.07}$ & $1.27_{-0.08}^{+0.09}$ & $0.93_{-0.08}^{+0.09}$ & $0.25_{-0.07}^{+0.07}$ & $0.17_{-0.06}^{+0.06}$ & $0.19_{-0.03}^{+0.03}$ & $0.17_{-0.03}^{+0.03}$ \\
\hline V347 Pav ....................... & $0.53_{-0.05}^{+0.06}$ & $0.63_{-0.08}^{+0.09}$ & $0.68_{-0.09}^{+0.10}$ & $0.87_{-0.02}^{+0.02}$ & $0.63_{-0.02}^{+0.02}$ & $0.60_{-0.07}^{+0.07}$ & $0.57_{-0.05}^{+0.05}$ \\
\hline 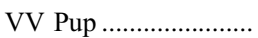 & $0.96_{-0.06}^{+0.06}$ & $1.11_{-0.09}^{+0.10}$ & $1.01_{-0.08}^{+0.09}$ & $0.67_{-0.02}^{+0.02}$ & $0.52_{-0.02}^{+0.02}$ & $0.34_{-0.05}^{+0.05}$ & $0.26_{-0.04}^{+0.04}$ \\
\hline MR Ser...................... & $3.71_{-0.09}^{+0.00}$ & $3.14_{-0.11}^{+0.11}$ & $3.04_{-0.11}^{+0.12}$ & $1.30_{-0.03}^{+0.03}$ & $1.44_{-0.04}^{+0.02}$ & $1.45_{-0.12}^{+0.05}$ & $1.17_{-0.07}^{+0.04}$ \\
\hline
\end{tabular}
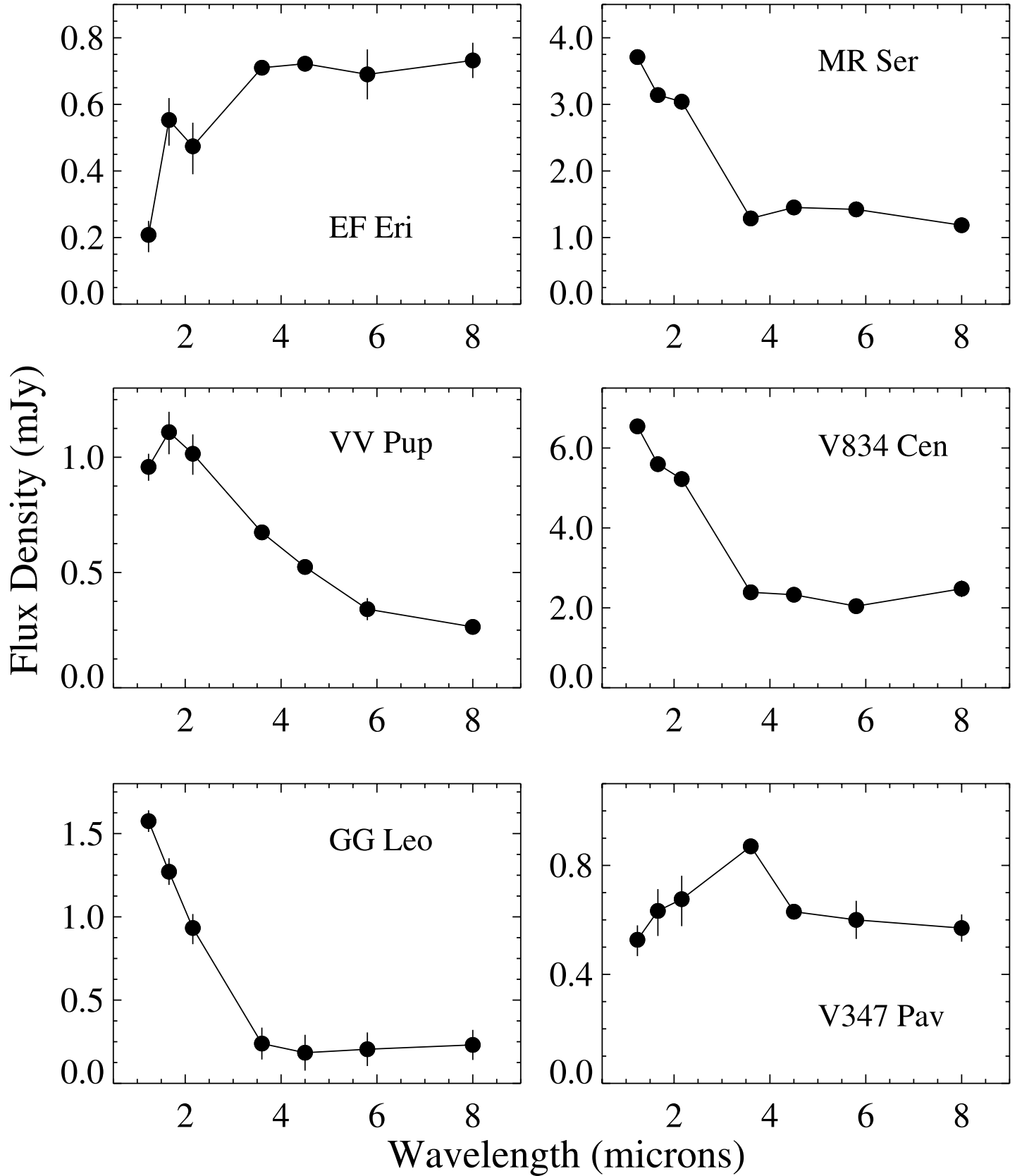

FIG. 1.-Observed SEDs for our six polars, from 2MASS to IRAC. 

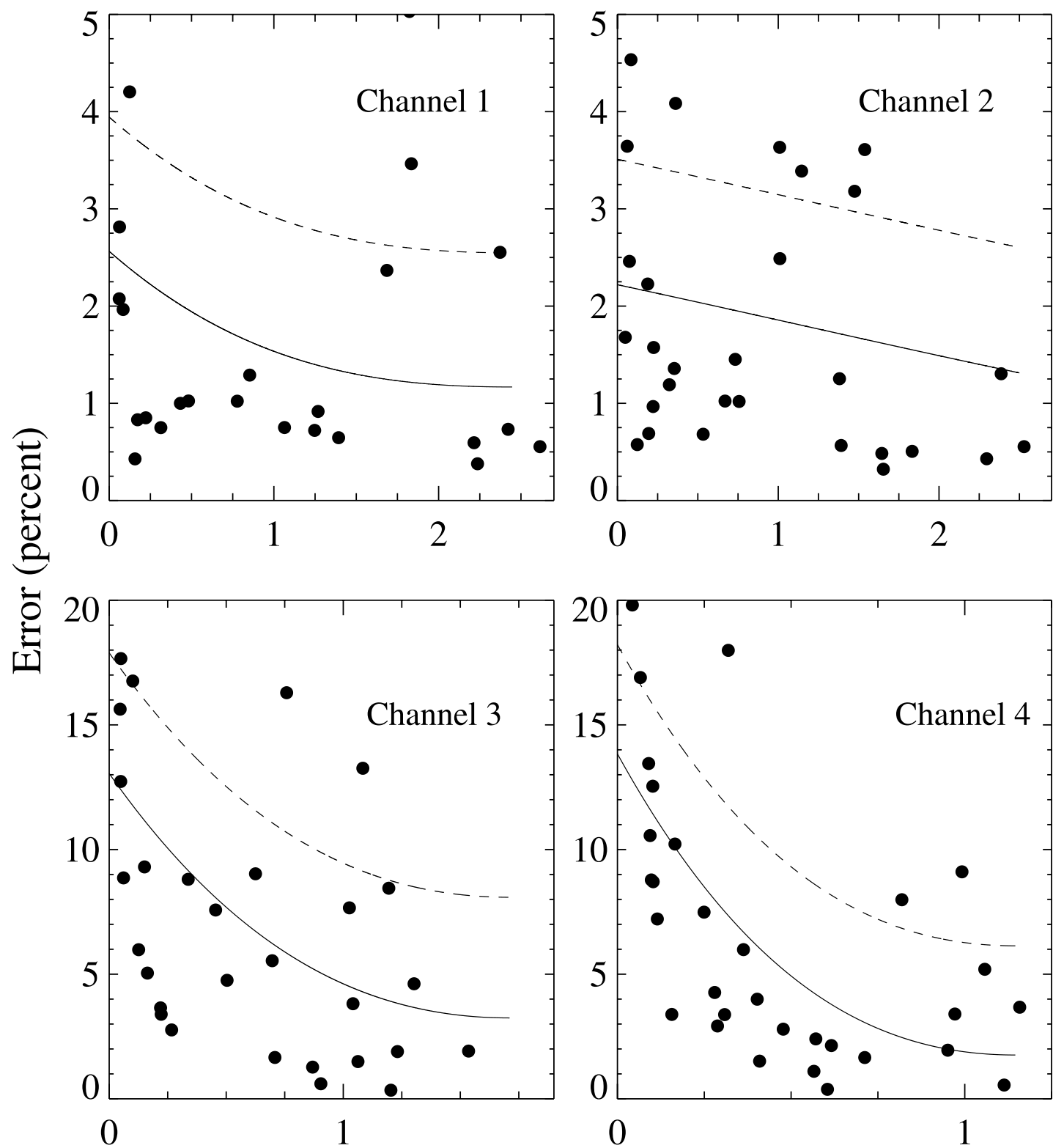

\section{Flux Density (mJy)}

FIG. 2.- Standard deviation of the flux densities in the five BCD frames vs. flux density measured in the mosaic for a number of isolated stars in our IRAC fields. The solid line is a best-fit polynomial to the data. The dashed line is best fit plus rms of residuals (i.e., the $1 \sigma$ uncertainty on the fit).

determine the relative errors for our data, which can then be adjusted in the future to incorporate the absolute errors as they become better known.

We performed aperture photometry on a number of isolated stars in each field (including fields for targets other than our polars), covering a wide range of flux densities. This was carried out for both the mosaicked images and the five individual BCD frames that were used to create each mosaic. The standard deviation of the five individual BCD flux density measurements of each test star was then plotted against the final flux density of the star measured from the mosaic (see Fig. 2), in the expectation that the standard deviation would decrease with increasing brightness. We find that there is indeed a correlation between the standard deviation and the final flux density, but there is considerable scatter around the best polynomial fit to the data (solid lines in
Fig. 2). To allow for this, we subtracted the polynomial fit from the data, calculated the standard deviation of the residuals, and added this to the best fit at each flux density to derive the total error estimate as a function of flux density for each IRAC channel (dashed lines in Fig. 2). We adopt these uncertainties for all future IRAC flux density measurements.

\section{INFRARED SPECTRAL ENERGY DISTRIBUTION MODELS}

In Paper I we briefly discussed the likely contributions to the IR SEDs of polars from a number of expected system components. In this paper we present detailed physical models to compare with our observed SEDs. The available model components are described below, and we discuss the modeling results in $\S 4$. 
TABLE 3

Polar System Parameters from the Literature

\begin{tabular}{|c|c|c|c|c|c|c|c|c|c|}
\hline POLAR & $\begin{array}{c}P_{\text {orb }} \\
\text { (minutes) }\end{array}$ & $\begin{array}{c}i \\
\text { (deg) }\end{array}$ & $\begin{array}{c}T_{\mathrm{WD}} \\
(1000 \mathrm{~K})\end{array}$ & $\begin{array}{c}\text { SECONDARY } \\
\text { SPECTRAL TyPE }\end{array}$ & $\begin{array}{l}M_{\mathrm{WD}} \\
\left(M_{\odot}\right)\end{array}$ & $\begin{array}{c}M_{2} \\
\left(M_{\odot}\right)\end{array}$ & $\begin{array}{l}\text { Distance } \\
(\mathrm{pc})\end{array}$ & \multicolumn{2}{|c|}{$B(\mathrm{MG})$} \\
\hline V834 Cen....... & $101.517[20]$ & $50(10)[17]$ & $14.3(0.9)[1]$ & M7.5-8.5 [10] & $0.66(18)[17]$ & $0.06[21]$ & $144_{-23}^{+18}[1]$ & $23[4]$ & \\
\hline EF Eri.................. & $81.022932(8)[11]$ & $55(5)[11]$ & $9.5(0.5)[2]$ & $\mathrm{M} 6+/ \mathrm{L} 4-5[2,7]$ & $0.6[2]$ & $0.055[9]$ & $42-120[2,7]$ & $13.8[9]$ & $16.5,21[5]$ \\
\hline GG Leo .......... & 79.87946(7) [3] & $50-60[3]$ & $8-12[3]$ & M5+ [3] & $1.13(3)[14]$ & $0.09[3]$ & $100-300[3]$ & $20-30[3]$ & \\
\hline V347 Pav ...... & $90.082219(52)[14]$ & $64-72[12]$ & $11.8_{-0.5}^{+0.6}[1]$ & $\ldots$ & $1.00(6)[14]$ & $0.16[13]$ & $171_{-38}^{+33}[1]$ & $\ldots$ & $15,20[12]$ \\
\hline VV Pup .......... & $100.436[20]$ & $78(5)[20]$ & $11.9_{-0.5}^{+0.6}[1]$ & M7 [8] & $0.73(5)[8]$ & $0.10(2)[8]$ & $129_{-21}^{+29}[8]$ & $\ldots$ & $32, \ldots[18]$ \\
\hline
\end{tabular}

NoтEs.-With the exception of distance and secondary star spectral type, the values of these parameters are held constant during our modeling. When there is a formal uncertainty or range of values listed here, the fixed value used in the models is the nominal or midpoint parameter value, respectively.

References.- (1) Araujo-Betancor et al. 2005; (2) Beuermann et al. 2000; (3) Burwitz et al. 1998; (4) Ferrario et al. 1992; (5) Ferrario et al. 1996; (6) Harrison et al. 2005; (7) Howell \& Ciardi 2001; (8) Howell et al. 2006a; (9) Howell et al. 2006b; (10) Mennickent et al. 2004; (11) Piirola et al. 1987; (12) Potter et al. 2000; (13) Ramsay et al. 1996; (14) Ramsay et al. 2004; (15) Schwope et al. 1991; (16) Schwope et al. 1993a; (17) Schwope et al. 1993b; (18) Wickramasinghe \& Meggitt 1982; (19) Wickramasinghe et al. 1991; (20) http://cvcat.net and references therein; (21) calculated from other parameters (see text).

\subsection{Initial Values of Model Parameters}

Our basic model includes several components (see below), each specified by a number of parameters (size, temperature, mass, etc.). We have held constant the values of as many as possible of these parameters using information obtained from various literature sources for each target (see Table 3). Known relationships between physical parameters were used to infer the values of additional parameters from an observationally determined one; for example, given a WD mass based on observational data, we used the mass-radius relation for a magnetic, carbon core WD with $\gamma=0.1$ from Suh \& Mathews (2000) to calculate the corresponding WD radius. Radii and temperatures corresponding to a given secondary star mass and spectral type were obtained from Cox (2000) and Pont et al. (2005). In the absence of measured physical parameters in the literature, substellar (i.e., L type) secondary stars were assumed to have $M_{2}=0.08 M_{\odot}, R_{2}=$ $0.1 R_{\odot}$, and $T_{2}=1500-2000 \mathrm{~K}$ (with higher temperature corresponding to earlier L spectral type; Kirkpatrick et al. 1999). In addition, parameters relating to the geometry of the CV (binary separation, Roche surfaces, etc.) were calculated via the well-known Roche potential relations (e.g., Kopal 1959, 1969, 1970) that depend primarily on component masses and the orbital period and can be solved numerically by utilizing an iterative approach (see Fig. 3).

The masses and radii of the secondary stars in our models are, to first approximation, consistent with filling their Roche lobes; however, whether or not the Roche lobe is filled is extremely sensitive to small changes in the mass or radius of both the WD and secondary star. We expect that the masses and radii of the component stars, which we have obtained from the literature or calculated as described above, are not exactly known. Consequently, we have generally not been overly concerned with ensuring that the model secondary stars precisely fill their calculated Roche lobes for a given CV system geometry. In a couple of cases, the secondary star spectral type required in the model that best reproduces the observed SED is not consistent with the mass estimates from the literature. In these cases, we suggest that either the mass estimates are inaccurate or we are encountering the well-known issue of the wide range of observed masses for each spectral type of CV secondary star (e.g., Smith \& Dhillon 1998).

\subsection{The White Dwarf}

The contribution of the WD primary star is simulated using a simple blackbody curve corresponding to the temperature of the $\mathrm{WD}$, scaled to the WD size and distance to the CV. Although, in detail, the SEDs of WDs are not identical to those of blackbodies, the falling Rayleigh-Jeans tail of the blackbody curve in the IR is a reasonable approximation to the long-wavelength SED of a WD. In any case, the largest WD contribution is typically only a few percent of the total observed flux density at the shortest IR wavelength (i.e., 2MASS $J$ band) and falls off rapidly to longer wavelengths.

\subsection{The Secondary Star}

The contribution of the secondary star is represented by choosing an empirical infrared SED template composed of $J H K_{s}$ (2MASS) and 3.6, 4.5, 5.8, and $8.0 \mu \mathrm{m}$ (IRAC) observations of a number of M, L, and T dwarfs (Patten et al. 2006). The Patten et al. (2006) sample includes stars of the following spectral types: M3.5, M5, M5.5, M6.5, M7, M8, M9, L0, L1, L2, L3, L4.5, L6,

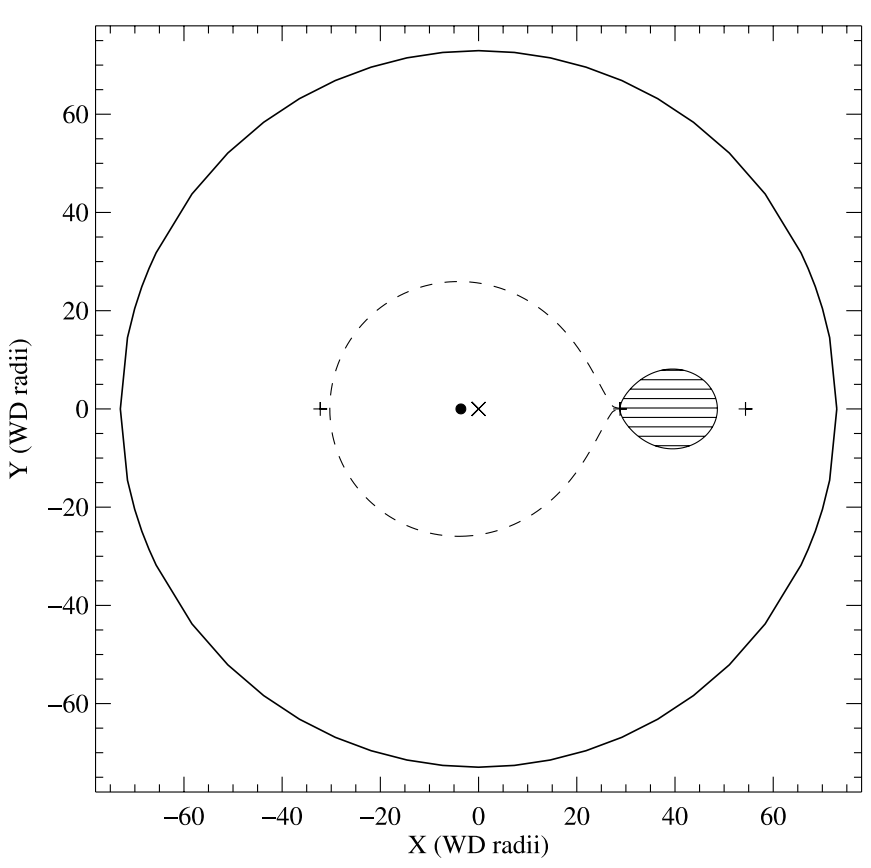

FIG. 3.- Sample model system geometry of EF Eri (face-on, to scale) for the model shown in Fig. $4 a$ and described in the text. The center of mass (marked with a cross) is at the origin, and the outer Lagrangian points are shown as plus signs. The WD is the small filled circle, and the secondary star is the large hashed area. The secondary star fills its Roche lobe; the Roche lobe of the WD is shown as a dashed line. The solid circle surrounding the CV is the inner edge of the circumbinary disk. 
L8, T0.5, T4.5, T5, T7, and T8. We caution that the sample includes only field stars, rather than stars in binaries that are subject to irradiation and mass transfer effects, and so may not exactly reliably reproduce the SED of a CV secondary star. In comparisons made, however, it has generally been shown that CV secondary stars do not deviate significantly from their isolated counterparts (e.g., Smith \& Dhillon 1998), and there is a long history of using field dwarfs to approximate the secondary stars in CVs. We also point out that no models of CV secondary stars exist at IRAC wavelengths, and therefore we use these templates as a first approximation. We compiled the distances to each of the template stars from various literature sources in order to transform all of their observed flux densities to a standard distance of $10 \mathrm{pc}$. Almost all of the template stars have distances measured via trigonometric parallax. The most distant template star is located at $\approx 27 \mathrm{pc}$, but $60 \%$ of them are closer than $10 \mathrm{pc}$.

We then performed a linear interpolation between adjacent observed spectral types in the M, L, and T groups, separately at each of the seven wavelengths, in order to "fill in" the SEDs of the spectral sequence in steps of 0.5 in spectral type. The observed data for spectral types of M5.5, L2, and T5 from the Patten et al. (2006) sample were not used in the interpolation process because they showed anomalous flux densities at all wavelengths compared to the relatively smooth transitions between adjacent spectral types seen for the remaining stars. Possible explanations for this include errors in the published distances or spectral type classifications for these stars, or they may possibly just be anomalous examples of their spectral type. We used interpolated SEDs to replace the observed SEDs for the three anomalous stars.

In all cases, Patten et al. (2006) estimate the uncertainties in the template flux densities as less than $2 \%$. Transformation to the standard distance will have increased this uncertainty by a few percent, up to $\approx 10 \%-15 \%$ in the worst case. All of our final model flux densities will be transformed to $d=10 \mathrm{pc}$ using the distances to each of the polars (either as a fixed, known value or as a free model parameter; see $\S 3.1$ ) in order to utilize these empirical low-mass stellar template SEDs as a model component.

\subsection{Cyclotron Emission}

As described in Paper I, we expect from a theoretical basis that cyclotron emission should be relatively unimportant as a contributor to the mid-IR SEDs of our polars, especially at the longest wavelengths. There is also empirical support for this claim. For example, Dhillon (1998) found "no evidence of cyclotron humps" in the $K$-band IR spectrum of MR Ser. The "worst-case" scenario occurs for EF Eri, which has the lowest magnetic field strength of our sample of polars, with the cyclotron fundamental occurring at $\lambda \approx 8 \mu \mathrm{m}$. Yet, Ferrario et al. (1996) found only very weak (amplitude $<1 \mathrm{mJy}$ ) cyclotron features at the red end of their 1-2.4 $\mu \mathrm{m}$ spectrum of EF Eri, a result that is also supported by the $K$-band spectra of Harrison et al. (2004), who found only a very weak cyclotron hump longward of $2 \mu \mathrm{m}$, with stronger features only at shorter wavelengths.

In order to include a model component representing cyclotron emission, we first calculated a representative profile as a function of wavelength. The shape of the cyclotron emission profile for a given field strength (which is a sum over harmonics) initially rises as $\nu^{2}$ before falling off rapidly with increasing frequency after the transition from optically thick to optically thin at some harmonic $1<m<10$ (Lamb \& Masters 1979; Cropper 1990; Frank et al. 2002). All of our polars except EF Eri have the cyclotron fundamental shortward of $\lambda \approx 4-6 \mu \mathrm{m}$, and we included the first 20 harmonics (which extends well into the optical regime in all cases). The corresponding profile of the cyclotron emission is strongly affected by a change in the transition harmonic from a value of $m=1$, but the difference in the observed profiles for values of $m \geq 2$ is relatively small. In general, the wavelength of the peak of the cyclotron emission component shifts shortward in proportion to $1 / m$ as $m$ increases. So, at small (large) values of $m$, there is more cyclotron emission at longer (shorter) wavelengths in the model SED. When $m \gtrsim 3$, we expect to see only the initial rising (toward shorter wavelength) powerlaw part of the cyclotron profile in the mid-IR SEDs of our polars. We investigate two cyclotron emission scenarios in our models, one in which the transition harmonic is at $m=1$ or 2 (i.e., approximately completely optically thin emission) and another in which $m \geq 3$ (i.e., partially optically thick emission).

Electrons approaching the WD experience a successively stronger magnetic field as their distance decreases. Thus, it is more appropriate to consider the cyclotron emission as a sum over emission components for a range of field strengths. In order to approximate this effect, we recalculated the cyclotron profile in $1 \mathrm{MG}$ steps from $1 \mathrm{MG}$ to the specified surface field strength. Each profile was scaled by the distance-dependent factor described in $\S 3.1$ of Paper I (to account for the change in electron density and temperature with distance from the WD). All of the profiles were then added together to obtain the final cyclotron emission profile for a given field. For the same surface field strength $(B)$, the "sum-over-field-strengths" prescription generally results in more cyclotron flux at longer wavelengths than in the single-field case. It also produces a cyclotron "pseudocontinuum," which tends to smooth over individual cyclotron humps (we already do not expect to see a strong signature of cyclotron humps in our model SEDs due to the poor spectral resolution of our data, which is matched in the model calculations).

The final parameter for the cyclotron component is a scale factor, which is multiplied with the model profile to match the observed flux densities. This model component is the only nonphysical component, in the sense that the absolute flux level is not predicted solely from other physical parameters (like temperature, distance, etc.). However, we are able to strongly constrain the scale factor for the cyclotron component because when it is combined with the WD and secondary star components, the total cannot exceed any of the observed flux densities.

\subsection{The Circumbinary Dust Disk}

The presence of a combination of optically thick and optically thin dust was first proposed by Berriman et al. (1985) as an explanation for the IR luminosity in CVs. Initial evidence for the likely presence of dust in several polars observed with Spitzer was described by us in Paper I. We now approach this analysis in more detail by including a realistic estimate of dust emission as a component in our SED model.

There are several possible origins for dust in CVs, including (1) remnant dust produced during the common envelope phase and ejected from the binary with some angular momentum, (2) dust lost from the accretion flow and ejected from the binary, (3) dust produced in the mass outflows during repeated nova or dwarf nova outbursts that does not escape from the gravity of the binary (e.g., Ciardi et al. 2006), and/or (4) dust produced over long timescales in the mass outflow of a wind from the accretion disk and/or WD (for further discussion of the possible origin of circumbinary dust in CVs see Taam \& Spruit 2001; Spruit \& Taam 2001; Taam et al. 2003; Dubus et al. 2004; Willems et al. 2005).

Dust that is located outside the Roche lobes of the stellar components in a CV should settle into a relatively cool, geometrically 
thin disk (Spruit \& Taam 2001; Taam et al. 2003; Belle et al. 2004; Dubus et al. 2004). There is a growing body of observational evidence for circumbinary dust disks in both post-AGB binary systems (Van Winckel 2004; De Ruyter et al. 2005, 2006) and pre-main-sequence binary stars (Hartmann et al. 2005). The detection of crystalline silicates in these circumbinary dust disks has been interpreted as an indicator of the long lifetimes of the disks (Hartmann et al. 2005; Deroo et al. 2006). As there is no previous observational evidence of circumbinary dust disks around CVs, it is impossible to say whether they have a similar longevity. However, the assumed temperature and density of the circumbinary disks are approximately an order of magnitude too low for significant sputtering of the grains (Burke \& Silk 1974), and if we assume that they are similar to those observed around other binary systems, then the dust disks will require little or no replenishment. The total minimum mass of dust that must be ejected from the system in order to form a circumbinary disk is therefore relatively small (approximately the mass of a large asteroid). This assumes that the dust is formed in the inner system prior to ejection, most likely in the outer atmosphere or wind of the low-mass secondary star (Lunine et al. 1989; Allard et al. 2001; Leggett et al. 2002; Tsuji 2002); the probability of the dust forming in situ in the disk itself is extremely low, as the dust formation mechanism requires high gas densities and, therefore, would require a high mass transfer rate from the inner binary system into the disk (Berriman et al. 1985).

Recently, Muno \& Mauerhan (2006) reported the discovery of a Spitzer IRAC mid-IR excess around several low-mass X-ray binaries (LMXBs), which they interpret as evidence for circumbinary dust disks with almost identical characteristics to the ones we model in this paper. Searches for dust disks around CVs have so far failed to uncover any direct evidence for their existence (e.g., Belle et al. 2004). In most CVs the circumbinary dust disk flux in the IR (if present) is likely to be overwhelmed by radiation from the cool outer regions of the accretion disk (or even the bright, early spectral type secondary star in long orbital period systems). In that regard, polars may be the only type of CV in which the flux contribution from cool dust can be detected. Despite the lack of direct observational evidence, the existence of circumbinary disks in CVs has been suggested as the explanation for both the high mass transfer rate in some systems and the observed lack of pileup of systems with orbital periods near the period minimum (Spruit \& Taam 2001; Taam \& Spruit 2001; Taam et al. 2003; Willems et al. 2005). However, as described in more detail in $\S 5$, the estimated total masses of our model circumbinary disks are likely too small to influence $\mathrm{CV}$ evolution as described in the papers cited above.

For inclusion as a component in our SED model, we initially make the assumption that the circumbinary dust disk is optically thick, as this presents a computationally simpler case and even (as described in Dubus et al. 2004) might be the most appropriate case for dust disks in CVs. For completeness, however, we also consider the case of an optically thin dust disk.

\subsubsection{The Optically Thick Case}

Our optically thick, geometrically thin dust disk model for the polars is based on the description of the expected properties of circumbinary disks in CVs given in Dubus et al. (2004). We do not consider the case for a warped or flared dust disk as our small number of data points cannot support the extra parameters required to model anything more complex than the geometrically thin case. We utilize the analytic solution for an optically thick dust disk radial temperature profile and corresponding integrated flux density derived in Jura (2003), Becklin et al. (2005), and ref- erences therein. In particular, equation (1) from Jura (2003) provides the temperature profile of the disk:

$$
T_{\mathrm{disk}} \approx\left(\frac{2}{3 \pi}\right)^{1 / 4}\left(\frac{R_{*}}{r}\right)^{3 / 4} T_{*},
$$

where $T_{*}$ and $R_{*}$ are the temperature and radius of the central irradiating star, respectively, and $T_{\text {disk }}$ is the resultant temperature of the disk at radial distance $r$. Equation (2) from Jura (2003) provides the integrated flux density, $F_{\text {disk }}$ :

$$
F_{\text {disk }}=\frac{2 \pi \cos i}{d^{2}} \int_{r_{\text {in }}}^{r_{\text {out }}} B_{\nu}\left(T_{\text {disk }}\right) r d r
$$

where $i$ and $d$ are the system inclination and distance, respectively, $r_{\text {in }}$ and $r_{\text {out }}$ are the inner and outer radii of the disk, and $B_{\nu}$ is the Planck function. In this formulation, the dust disk is assumed to be optically thick but geometrically thin (i.e., with negligible height), so the integrated flux density originates only from the visible surface of the disk (with no contribution from the negligibly tall disk rim).

In principle, the optically thick dust model is not sensitive to the exact properties of the dust grains (size, composition, etc.), as long as the grains are assumed to uniformly (re)radiate as blackbodies. The exponent on the radius dependence in the equation for the disk radial temperature profile, however, does have some implication for the assumed properties of the dust grains. A large exponent leads to lower temperatures near the inner edge of the disk, which corresponds to large dust grains that cool efficiently. Conversely, a small exponent leads to higher temperatures near the outer edge of the disk, which corresponds to small dust grains that do not cool efficiently. In practice, we have found that an exponent of $\frac{3}{4}$, as used in equation (1), is the only value that produces viable results. Larger exponents produce disk SEDs that rise too shallowly at longer wavelengths and are, overall, too faint to match the observed mid-IR flux densities without arbitrarily increasing the temperature of the disk's inner edge far beyond what could be produced by the irradiating stars. Smaller exponents produce disk SEDs that rise too steeply and are, overall, too bright to reproduce the observed SEDs without arbitrarily increasing the distances to the CVs to many hundreds or thousands of parsecs. Departure from an exponent of $\frac{3}{4}$ implies nonblackbody grains, which are further discussed in $\S 3.5 .2$.

The disks considered by Jura (2003) and Becklin et al. (2005) are around isolated WDs (G29-38 and GD 362, respectively). There are a number of differences that must be considered when applying this model to a circumbinary disk in a CV system. First, the disk will be centered around the center of mass of the CV instead of the WD. In the case of a low-mass (potentially substellar) secondary star, the difference between the center of the WD and the center of mass of the $\mathrm{CV}$ is relatively small ( $\leqslant 10 \%$ of the binary separation). Second, the inner edge of the disk will be much farther from the WD. In the WD disk models, the inner edge of the disk is typically located at a few to $\sim 10 \mathrm{WD}$ radii. However, in the case of a circumbinary disk, the inner edge is, by definition, located outside the Roche lobes of the stellar components. We assume an inner radius for the circumbinary disk equal to the tidal truncation radius at 1.7 times the separation of the stellar components in the CV (Dubus et al. 2004). For our short orbital period polars, this typically corresponds to $r_{\text {in }} \gtrsim 100 R_{\text {WD }}$. The outer edge of the disk is calculated to correspond to a temperature of $20 \mathrm{~K}$ using equation (1). This typically corresponds to $\sim(0.5-2) \times 10^{4} \mathrm{WD}$ radii for our polars, but in reality, the radius 
of the outer disk has a negligible effect on the model flux densities at the IRAC wavelengths and serves only as a conservative, consistent cutoff value. Third, we must consider irradiation heating of the disk from not only the WD but also the secondary star. ${ }^{3}$ For example, for a typical short-period CV composed of a WD with temperature $T_{\mathrm{WD}}=10,000 \mathrm{~K}$ and radius $R_{\mathrm{WD}}=0.01 R_{\odot}$ and a low-mass secondary star with $T_{2}=3000 \mathrm{~K}$ (i.e., $0.3 T_{\mathrm{WD}}$ ) and radius $R_{2}=0.1 R_{\odot}$ (i.e., $10 R_{\mathrm{WD}}$ ), the ratio of total luminosities (given by $L \propto R^{2} T^{4}$ ) is $L_{2} / L_{\mathrm{WD}}=0.8$. Thus, the total luminosity of the secondary star is comparable to that of the WD.

Unfortunately, the additional contribution from the secondary star makes our consideration of the heating of the circumbinary disk more complex because the secondary star is much farther from the CV's center of mass (i.e., the center of the circumbinary disk) than the WD and so is, at any instant, closer to the inner edge of the circumbinary disk. However, under the assumptions that the disk inner edge radius is large compared to the binary separation (see above) and that the orbital period is short (so, on a time-averaged basis, all parts of the disk "see" the secondary star equally), we neglect the offset of the secondary star and assume that all disk irradiating sources are located at the center of mass.

Using the typical $\mathrm{CV}$ parameters listed above with $P_{\text {orb }}=$ 90 minutes and stellar component masses of $M_{\mathrm{WD}}=0.6 M_{\odot}$ and $M_{2}=0.1 M_{\odot}$, the circumbinary disk will have an inner edge radius of $R_{\mathrm{in}}=100 R_{\mathrm{WD}}$. Assuming only heating from the $\mathrm{WD}$, equation (1) predicts an inner edge temperature of $T_{\text {in }} \approx 200 \mathrm{~K}$. If only the secondary star is considered as the heating source, then we get $T_{\text {in }} \approx 350 \mathrm{~K}$. We have considered two methods for estimating the temperature of the circumbinary disk's inner edge that is likely to result from heating by both stellar components in the $\mathrm{CV}$. The first method is to simply add together the inner edge temperatures predicted by heating from each of the stellar components individually. This would give $T_{\text {in }} \approx 550 \mathrm{~K}$ for the typical CV parameters described above. The second method is to calculate a single "equivalent" star with radius $R_{\text {equiv }}$ that is equal to the quadrature sum of $R_{\mathrm{WD}}$ and $R_{2}$ and temperature $T_{\text {equiv }}$ such that $L_{\text {equiv }}=R_{\text {equiv }}^{2} T_{\text {equiv }}^{4}$ is equal to the sum of the individual luminosities of the WD and secondary star. For the system parameters listed above, this gives $T_{\text {in }} \approx 450 \mathrm{~K}$. Given the uncertainty in estimating the likely heating effect of both stars and the fact that both of our suggested methods yield similar temperatures, we have used the circumbinary disk inner temperature as a free parameter in our model SED calculations. However, we have constrained $T_{\text {in }}$ to be within approximately $\pm 200 \mathrm{~K}$ of the value predicted for heating by a single equivalent star (i.e., the second method described above).

\subsubsection{The Optically Thin Case}

The basic structure of an optically thin circumbinary dust disk is identical to that in the optically thick case: it is geometrically thin and heated by irradiation from both stellar components, both of which are assumed to be located at the system center of mass. Our model is based on two main assumptions: first, that each dust particle (re)radiates as a blackbody, and second, that all of the particles are "seen" by the observer (i.e., there is no eclipsing of the disk by the stellar components and every one of the grains contributes to the total flux density). The former condition is not

\footnotetext{
${ }^{3}$ Fortunately, since polars lack an accretion disk, we do not have to consider this additional potential irradiating source. In principle, the accretion spot(s) at the WD magnetic pole(s) could be considered as additional discrete sources of irradiation; however, unless the magnetic axis is aligned very close to the WD equator, the geometrically thin circumbinary disk will not "see" the geometrically small accretion spot(s).
}

an unreasonable assumption since the inner disk radius is set at the tidal truncation radius, well away from the stellar components. We use the same disk temperature profile from Jura (2003) and Becklin et al. (2005) as we use in the optically thick case, with an outer disk radius corresponding to an outer disk temperature of $T_{\text {out }}=20 \mathrm{~K}$, also as used in the optically thick case. We assume that the disk has a constant volume density throughout, so the volume of material at any temperature (radius) in the disk increases with falling temperature (increasing radius). We used a fixed grain radius of $10 \mu \mathrm{m}$, which was suggested by Jura (2003) as the minimum grain size for dust debris disks around mainsequence stars, and a fixed dust grain density of $3 \mathrm{~g} \mathrm{~cm}^{-3}$. In fact, the exact values of the dust grain parameters that we use have little effect on the resultant model SED of the dust disk; for example, if we change the grain size (thereby reducing the available radiating surface area in the disk), then the required luminosity to match the observed SED can still be achieved by changing one of the other disk parameters, such as the volume density of the dust.

In all cases, we kept the disk thickness and volume density as low as possible to ensure that the disk remained optically thin; however, this was very difficult to quantify. The optical depth of the dust is given by

$$
\tau=\kappa_{s} \rho S
$$

where $\kappa_{s}$ is the mass absorption coefficient, $\rho$ is the volume density of the dust, and $S$ is the column length along the line of sight. The mass absorption coefficient is a function of dust composition, grain size and shape, dust temperature, and the frequency of the incident radiation. As a result, it is usually quoted in a frequency-dependent form, $\kappa_{\nu}$. It is highly temperature sensitive but extremely poorly studied for temperatures above $\sim 300 \mathrm{~K}$, as all of the literature on this subject deals with the cool interstellar medium and there are no published values of $\kappa_{\nu}$ at the temperatures found in our disks. Even the theoretically calculated values for the relatively well-studied low temperatures vary by a factor of 30, from 0.002 to $0.10 \mathrm{~cm}^{2} \mathrm{~g}^{-1}$ (Draine 1989). As a result, we have no certain way of testing the optical depth of our disks, but we adopted a simple, generic relation in an attempt to quantify our values:

$$
\kappa_{\nu}=0.1\left(\nu / 10^{12} \mathrm{~Hz}\right)^{\beta} \mathrm{cm}^{2} \mathrm{~g}^{-1},
$$

which is the general form given in Beckwith et al. (1990). The value of $\beta$ depends on the growth shape of the grains: spheroidal grains will have a higher value of $\beta$ than fractal ones (for an indepth discussion of these effects see Beckwith et al. 1990). We follow the example of Beckwith et al. (1990) and accommodate the uncertainty in the grain shape by adopting the general relation given above for $\kappa_{\nu}$ with $\beta=1$. In any case, the volume density, and therefore the optical depth, of the disk is dependent on the disk temperature profile, the grain size and mass density, and the inner and outer radii of the disk. Any calculation of the true optical depth of the disk requires better constraints on all of these parameters.

When the presence of dust can explain the long-wavelength end of a polar SED, we generally have been unable to differentiate between the optically thick and thin circumbinary dust disks: because of the number of available parameters used to describe each type of disk, we can produce qualitatively similar model SEDs using either type of disk. Thus, in the remainder of this paper we generally only discuss the less complex optically thick 
case, with the exception that we consider an estimate of the total dust mass from the optically thin case (see $\S 5$ ).

\section{MODELING RESULTS}

We emphasize that we have seven data points in each of our SEDs, only four of which (from IRAC) are in the mid-IR. Consequently, our modeling results are intended to illustrate general trends in polars as a class rather than provide a specific, detailed representation of each of our target polars. In general, we started each model by including best estimates for the WD and secondary star components, then attempted to fit the observed $8 \mu \mathrm{m}$ flux density through the addition of either cyclotron or dust emission, without exceeding the observed flux density at any shorter wavelength. In reality, these systems could (and likely do) include contributions from both cyclotron and dust emission, but we have too few data points to constrain such a complex model in any meaningful way. However, as described in detail in the following sections, success at reproducing the observed $8 \mu \mathrm{m}$ point with a particular model configuration while simultaneously not exceeding the observed flux densities at shorter wavelengths can allow us to rule out cyclotron emission (often) or dust (occasionally) as a dominant contributor at the long-wavelength end of the observed SEDs.

Because of the relatively small number of observational constraints compared to the degrees of freedom in the models, optimization of model parameters was accomplished via manual testing. In general, the values of the model parameters can be varied within a range of about $\pm 5 \%-10 \%$ and will still produce a model SED of comparable quality to the "best" cases discussed below. For convenience in discussing our results, we divided the targets into three classes based on observed SED morphology. Class I contains EF Eri and V347 Pav, which have SEDs in which the IRAC portion is brighter than the 2MASS portion. Class II contains VV Pup, whose SED is most similar in shape to a falling Rayleigh-Jeans-like tail and may be dominated by the secondary star. Class III contains V834 Cen, GG Leo, and MR Ser, which have SEDs in which the 2MASS portion is bright and falls steeply toward long wavelengths but the IRAC portion is relatively flat. We now discuss our modeling results for the polars in each of these classes.

\subsection{Class I: Bright IRAC}

The two polars in this class have oddly shaped SEDs compared to the other four polars in our sample. Specifically, the IRAC portion of the SED, while still approximately flat at the longest wavelengths, is brighter than the 2MASS portion (see Fig. 1). This possibly stems from another potential complication to the interpretation and modeling of our polar SEDs, namely, that polars are highly variable objects. Their brightness changes dramatically on irregularly spaced long timescales (weeks to years) between high and low accretion states (by up to a few magnitudes) and over their orbital period (by as much as $2 \mathrm{mag}$ ). In order to best compare the nonsimultaneous 2MASS and IRAC measurements for our targets, we attempted to determine both the accretion state and orbital phase of each of the polars at the times of both sets of measurements.

We used flux measurements from the literature and long-term light curves from the American Association of Variable Star Observers (AAVSO) to check the accretion state of the polars. Of our six targets, we were only able to definitively confirm that one (EF Eri) was in the same accretion state (low) during both the 2MASS and Spitzer observations. We suspect that at least three of the systems ( V834 Cen, GG Leo, and MR Ser, i.e., the class III objects) were in high accretion states during the 2MASS observations because of the steepness of the 2MASS portion of their SEDs, which may be indicative of the falling Rayleigh-Jeans-like tail of accretion-generated luminosity. The 2MASS-IRAC SED transition in VV Pup (i.e., the class II object) is very smooth, which suggests that the data were obtained during the same accretion state (regardless of which state). Light curves from the AAVSO show that VV Pup was in a high state for the 2MASS observations. There is no ground-based coverage at the time of the Spitzer observations, but from the long-term light curve, it is obvious that the system spends most of its time in a high state, and the closest data points show no indication of the system dropping into a low state at that time.

So far, there are no published orbital light curves of polars in the mid-IR, so we have no evidence that the flux levels at the IRAC wavelengths vary with the same phasing and/or amplitude as in the optical or 2MASS wave bands, but for completeness we consider this possibility. We used published ephemerides to determine the orbital phasing of the 2MASS and IRAC data sets for our targets and then used published optical or near-IR light curves to estimate whether the orbital brightness states of those data sets were comparable. Both EF Eri and V347 Pav were observed by 2MASS and Spitzer at significantly different orbital phases that correspond to different orbital brightness states (in both cases, the 2MASS data were obtained during a faint state, and the IRAC data during a bright state). The other four targets were observed by both 2MASS and Spitzer at similar orbital phases that correspond to the same orbital brightness state, and so the results at all wavelengths should be directly comparable. In the absence of mid-IR orbital light curves, we have assumed that the ratio of bright to faint flux density for the IRAC data is the same as observed in the optical/near-IR. In any case, there are enough model parameters to compensate for different bright/faint scale factors (and, of course, if the mid-IR is shown in the future not to vary over the orbital period like the optical/near-IR, then this contingency can be discarded!). We note that even after attempting a scaling correction, the SEDs for EF Eri and V347 Pav are morphologically similar to each other but different from the other classes we have defined (see $\S \S 4.1 .1$ and 4.1.2 for details). They show flat IRAC SEDs like class III, but the 2MASS portions of their SEDs resemble class II.

\subsubsection{EF Eridani}

We selected EF Eri for our IRAC campaign due, in large part, to its extremely red $\left(J-K_{s}>2.5\right)$ 2MASS colors (Hoard et al. 2002), which exceed even the reddest IR colors for mid-L dwarfs (Leggett et al. 2002). This CV was discovered as an X-ray source by Gursky et al. (1978) and subsequently identified by Griffiths et al. (1979) and Williams et al. (1979) as a probable AM Her type. The magnetic nature of the system was confirmed by Tapia (1979) from measurements of the linear and circular polarization, which he used to derive the very short orbital period of $P_{\text {orb }} \approx 81$ minutes, which was later refined by Piirola et al. (1987).

EF Eri entered an extended low state in 1997, only emerging back into a high state in 2006 March, and only remaining bright for a few weeks before dropping back into the low state. The low-state optical spectrum obtained by Wheatley \& Ramsay (1998) displayed only weak line and cyclotron emission, indicating that the low-state mass transfer rate is extremely small. Modeling of this spectrum by Beuermann et al. (2000) set a limit of M9 V or later for the secondary star spectral type and a distance estimate of $d=100 \pm 20 \mathrm{pc}$ (consistent with the upper limit of $d=119$ pc estimated from near-IR observations by Young $\&$ 


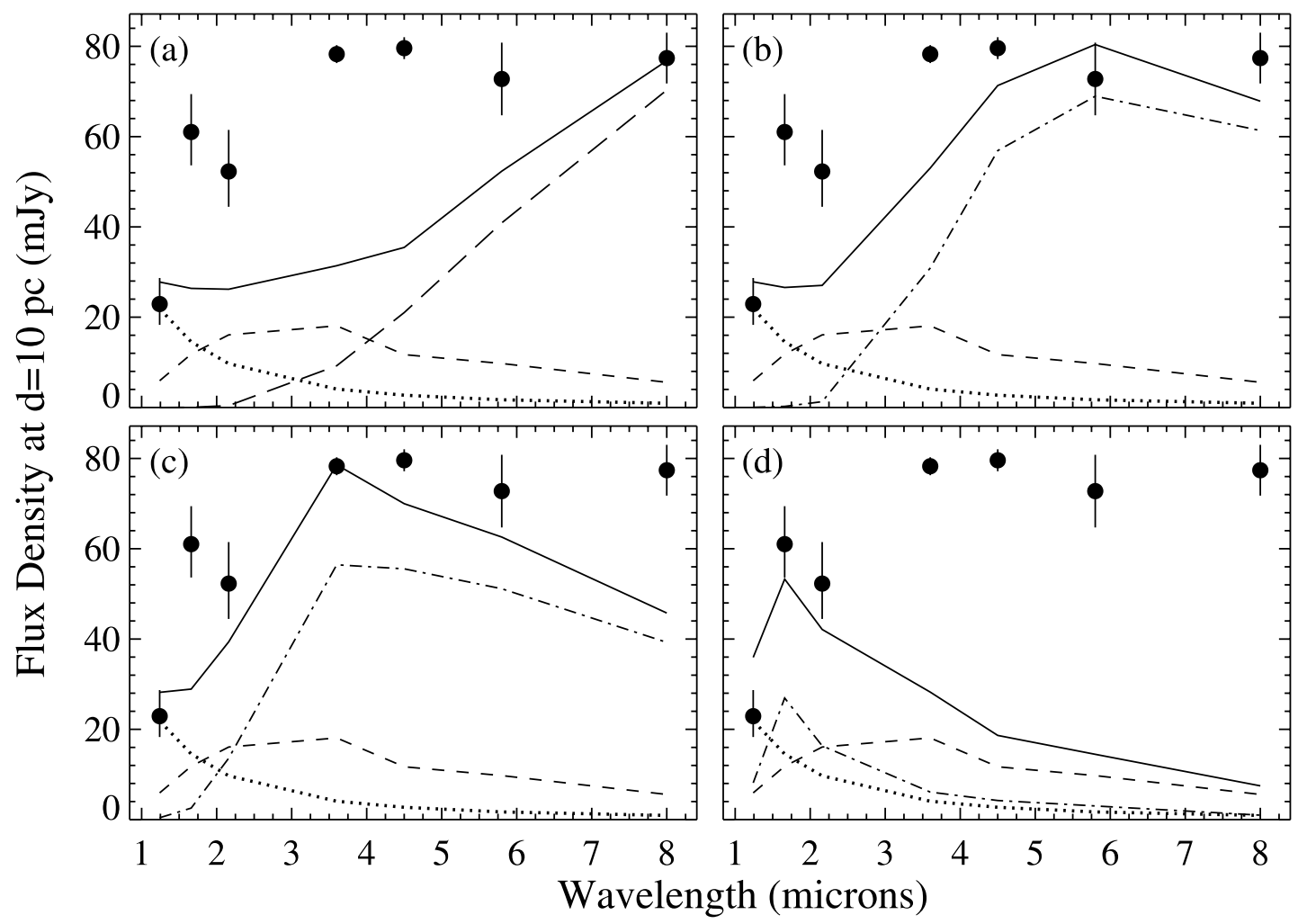

FIG. 4.- Representative model SEDs for EF Eri showing the observed data ( filled circles), total model SED (solid line), and model components: the WD (dotted line), the secondary star (short-dashed line), a circumbinary dust disk (long-dashed line), and cyclotron emission (dot-dashed line). (a) Circumbinary disk model; (b) cyclotron model with $m=2 ;(c)$ cyclotron model with $m=3 ;(d)$ single-field cyclotron model with $m=5$. See text and Table 4 for details.

Schneider 1981). Howell \& Ciardi (2001) detected the secondary star in $K$-band spectra with a spectral type of either M6 V or L4-5, although it is impossible to tell whether the star has dropped below the hydrogen-burning limit based solely on spectral type, as the temperatures may depend on irradiation and evolutionary history. Harrison et al. (2003) presented simultaneous multiwavelength optical and infrared photometry in which they believed they detected a brown dwarf secondary star in the $H$ and $K$ bands, but follow-up phase-resolved spectroscopy (Harrison et al. 2004) showed that the variability in both bands is dominated by cyclotron emission throughout the orbital cycle, most likely originating at both of the WD magnetic poles.

Recently, Howell et al. (2006b) determined an extremely low mass for the secondary star in EF Eri $\left(M_{2}=0.055 M_{\odot}\right)$, suggesting that an L spectral type classification is, indeed, appropriate. At a distance of $d \approx 100 \mathrm{pc}$, the secondary star in EF Eri cannot be earlier than L5.0, or the combined flux densities of just the WD and secondary star will significantly exceed the observed $J$-band point. Our model for EF Eri uses the WD parameters from Beuermann et al. (2000) and the secondary star parameters from Howell et al. (2006b) and assumes a spectral type of L5.0 for the secondary star (see Table 3). We then combined this model with an additional component representing cyclotron or dust emission to explore the limiting cases for the ability of either mechanism to explain the observed long-wavelength SED of EF Eri (without exceeding the short-wavelength end).

Figure 4 shows representative model SEDs for EF Eri. The model containing a circumbinary dust disk (Fig. $4 a$ and model 1 in Table 4) can easily reproduce the observed $8 \mu \mathrm{m}$ point but significantly underestimates the observed flux densities at the shorter wavelengths (except at the shortest wavelength, $J$ band). When the cyclotron transition harmonic is set to $m=1$, the cyclotron emission is essentially indistinguishable from the circumbinary disk component, so, in the case of completely optically thin cyclotron emission, we cannot differentiate between cyclotron or dust producing the $8 \mu \mathrm{m}$ emission. However, neither mechanism (on its own) can produce the high levels of emission at shorter wavelengths without greatly exceeding the $8 \mu \mathrm{m}$ point. We found this to be true in all of our polars, so we do not mention it again in the discussion of our modeling results and defer discussion of completely optically thin cyclotron emission to $\S 5$.

Figure $4 b$ (see model 2 in Table 4 ) shows the model SED having a cyclotron component with transition harmonic at $m=2$, which best reproduces the observed long-wavelength end of the SED but still underestimates both the short-wavelength end and the $8 \mu \mathrm{m}$ point (even when the model $5.8 \mu \mathrm{m}$ flux density is pushed to the upper uncertainty limit of the observed point at that wavelength, as shown in the figure). Switching the cyclotron component to increasingly optically thick configurations (e.g., $m=3$; see Fig. $4 c$ and model 3 in Table 4 ) recovers some of the short-wavelength flux, but at the expense of substantially underestimating the IRAC portion of the SED.

Up to now, we were using the sum-over-field-strengths prescription for the cyclotron emission (see $\S 3.4$ ). As shown in Figure $4 d$ (model 4 in Table 4), the single field strength approach can approximately reproduce the bright observed $H$-band point (at the expense of overestimating the $J$-band point) because the cyclotron humps are not blended together into a pseudocontinuum as in the sum-over-field-strengths approach. It is possible that if the intensity scaling of profiles produced farther from the 
TABLE 4

Model Parameters for EF Eri

\begin{tabular}{|c|c|c|c|c|c|c|c|}
\hline Component & Parameter $^{\mathrm{a}}$ & Model 1 & Model 2 & Model 3 & Model 4 & Model $5^{\mathrm{b}}$ & Model $6^{\mathrm{b}}$ \\
\hline System............................. & $d(\mathrm{pc})$ & 105 & 105 & 105 & 105 & 105 & 105 \\
\hline \multirow[t]{2}{*}{ SS } & $T_{2}(\mathrm{~K})$ & 1700 & 1700 & 1700 & 1700 & 1790 & 1790 \\
\hline & Spectral type & L5.0 & L5.0 & L5.0 & L5.0 & L3.5 & L3.5 \\
\hline \multirow[t]{5}{*}{ CYC } & $B(\mathrm{MG})$ & $\ldots$ & 13.8 & 13.8 & 13.8 & $\ldots$ & 13.8 \\
\hline & $m$ & $\cdots$ & 2 & 3 & 5 & $\cdots$ & 2 \\
\hline & Sum over fields? & $\ldots$ & $\mathrm{Y}$ & $\mathrm{Y}$ & $\mathrm{N}$ & $\ldots$ & $\mathrm{Y}$ \\
\hline & $f_{\nu, \mathrm{CYC}} / f_{\nu, \mathrm{SS}}(3.6 \mu \mathrm{m})$ & $\ldots$ & 1.71 & 3.12 & 0.34 & $\ldots$ & 1.55 \\
\hline & $f_{\nu, \mathrm{CYC}} / f_{\nu, \mathrm{SS}}(8.0 \mu \mathrm{m})$ & $\ldots$ & 11.01 & 7.04 & 0.18 & $\ldots$ & 9.34 \\
\hline \multirow[t]{4}{*}{ CBD } & $r_{\text {in }}$ & $73 R_{\mathrm{WD}}$ & $\ldots$ & $\ldots$ & $\ldots$ & $73 R_{\mathrm{WD}}$ & $\ldots$ \\
\hline & $T_{\text {in }}(\mathrm{K})$ & 665 & $\ldots$ & $\ldots$ & $\ldots$ & 665 & $\ldots$ \\
\hline & $r_{\text {out }}$ & $7800 R_{\mathrm{WD}}$ & $\ldots$ & $\ldots$ & $\ldots$ & $7800 R_{\mathrm{WD}}$ & $\ldots$ \\
\hline & $T_{\text {out }}(\mathrm{K})$ & 20 & $\ldots$ & $\ldots$ & $\ldots$ & 20 & $\ldots$ \\
\hline
\end{tabular}

${ }^{\text {a }}$ Fixed model parameter values for this target include $R_{\mathrm{WD}}=0.0125 R_{\odot}$ and $R_{2}=0.1 R_{\odot}$. See Table 3 for the values of other parameters not listed here.

b These models refer to the scaled 2MASS data; see text.

WD (i.e., at smaller magnetic field strengths) falls off more steeply than estimated in Paper I, then the final profile might be dominated by the emission produced very near the WD surface and, hence, retain the sharply peaked shape seen in Figure $4 d$. A better reproduction of the EF Eri SED might be achieved through a combination of models similar to models 1 and 4 (i.e., the SEDs shown in Figs. $4 a$ and $4 d$ ). However, even in this case, there would be some "missing" flux at 3.6 and $4.5 \mu \mathrm{m}$, suggesting the need for either model components with different spectral profiles or an additional emitting system component. Adding more model parameters/components is not warranted by the ability of these sparse data to constrain complex models. We note, however, that some experimentation demonstrated to us that a simple blackbody component with $T \approx 1000 \mathrm{~K}$ produces an emission profile that peaks around $4.5 \mu \mathrm{m}$ and could fill in the EF Eri model SED as required.

As noted in $\S 4.1$, the 2MASS and IRAC data for EF Eri were obtained during different orbital brightness states (faint and bright, respectively). The low-state $K$-band photometric light curves of Harrison et al. (2003) show that $F_{\text {bright }} / F_{\text {faint }} \approx 1.67$. Assuming that this ratio holds into the mid-IR, we scaled the 2MASS data for EF Eri by a factor of 1.67 to match the short-wavelength portion of the SED with the IRAC data and repeated our modeling trials. We scaled the 2MASS data instead of the IRAC data in order to avoid altering the observed value of the $8 \mu \mathrm{m}$ flux density (which is the main target of our modeling efforts). Modeling these scaled data yields substantially similar results to those described above for the unscaled data (e.g., Fig. 5 and models 5 and 6 in Table 4). The model parameters must be changed slightly from the unscaled versions to account for the difference in the scaled flux density levels; notably, the secondary star spectral type can be as early as L3.5 without exceeding the scaled $J$-band point (at $d=105 \mathrm{pc}$ ). Regardless, the general trends seen in the unscaled models are preserved in the scaled models.

\subsubsection{V347 Pavonis}

V347 Pav was discovered by Pounds et al. (1993) in the Röntgensatellit (ROSAT) Wide Field Camera survey. It was identified as a CV by O'Donoghue et al. (1993) and confirmed as an AM Her type by Bailey et al. (1995). O'Donoghue et al. (1993) showed that the optical light curve is complex, with variability over the orbital cycle of approximately $1 \mathrm{mag}$, and displaying substantial variations from one orbital cycle to the next. Their photometry and spectroscopy both suggested an orbital period of $P_{\text {orb }} \sim 90$ minutes, which was later refined by Ramsay et al. (2004) to $P_{\text {orb }}=90.082219(52)$ minutes.

Bailey et al. (1995) fit the optical circular polarization and light intensity curves with two linearly extended cyclotron emission regions on the surface of the WD, with a model polar field

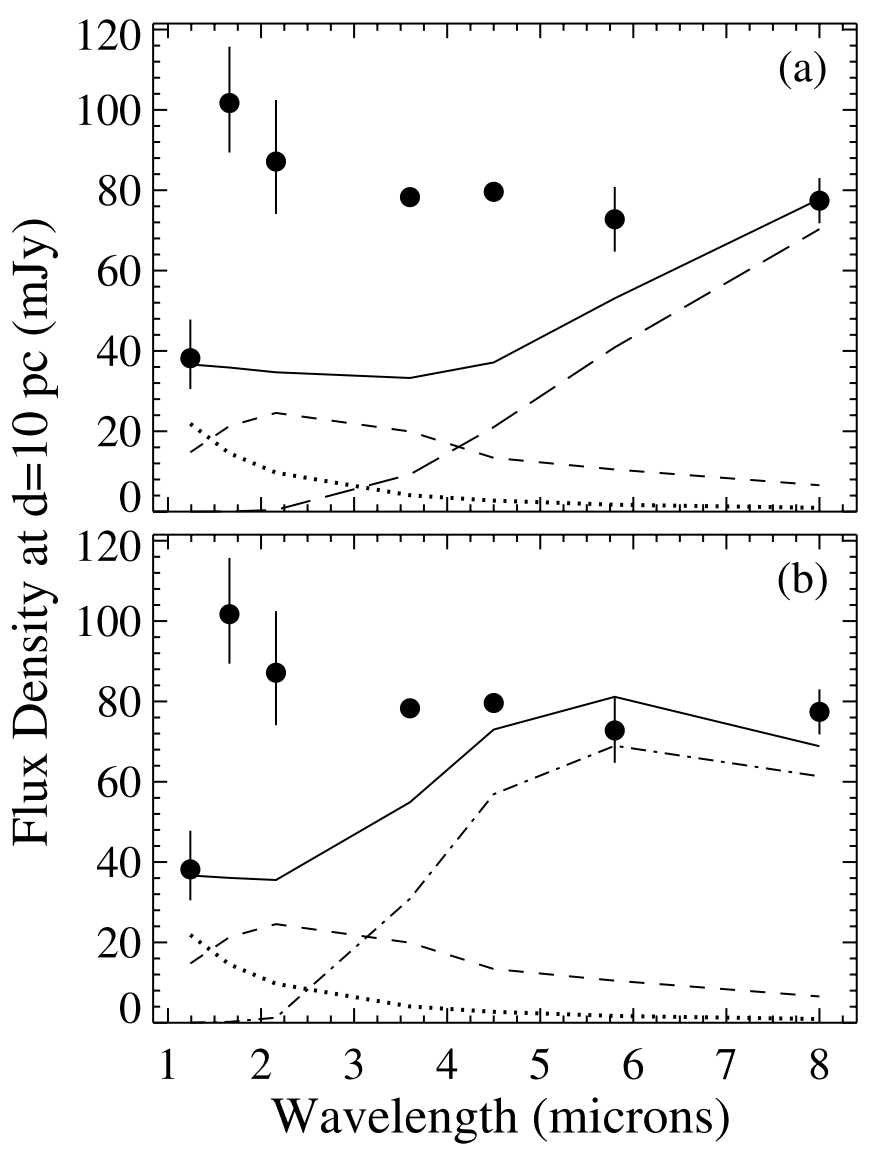

FIG. 5.- Same as in Fig. 4, but with the 2MASS data for EF Eri scaled by a factor of 1.67 to account for potential orbital brightness variations. (a) Circumbinary disk model; $(b)$ cyclotron model with $m=2$. See text and Table 4 for details. 


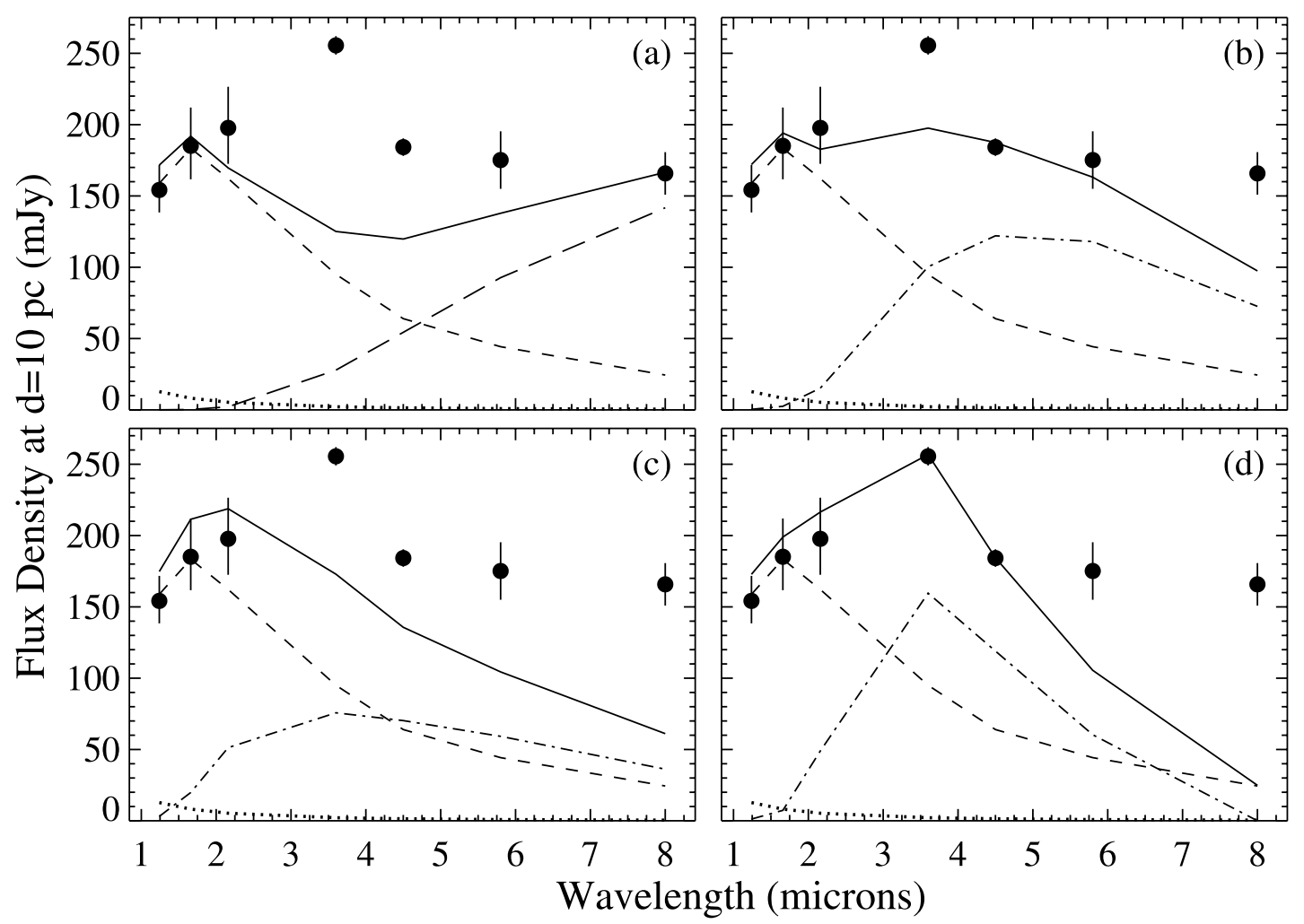

FIG. 6.- - Representative model SEDs for V347 Pav showing the observed data ( filled circles), total model SED (solid line), and model components: the WD (dotted line), the secondary star (short-dashed line), a circumbinary dust disk (long-dashed line), and cyclotron emission (dot-dashed line). (a) Circumbinary disk model; (b) cyclotron model with $m=2 ;(c)$ cyclotron model with $m=3 ;(d)$ single-field cyclotron model with $m=2$. See text and Table 5 for details.

strength of $B=25 \mathrm{MG}$ and an orbital inclination of $i=60^{\circ}$. This was constrained further by Ramsay et al. (1996), who used the orbital period-secondary star mass relation for a mainsequence star to predict $M_{2}=0.16 M_{\odot}{ }^{4}{ }^{4}$ which, combined with the fact that they had failed to observe any eclipses of the system, allowed them to put an upper limit on the inclination of $i<78^{\circ}$. Subsequent Stokes imaging by Potter et al. (2000) constrained the inclination more closely to $64^{\circ}<i<72^{\circ}$, with magnetic field strengths of $B=15$ and $20 \mathrm{MG}$ for the upper and lower accretion poles, respectively.

At the nominal literature distance of $d=171 \mathrm{pc}$, the secondary star can be no earlier than spectral type M6.0, or the combined secondary star plus WD (the latter contributes less than $8 \%$ of the observed SED at all wavelengths) exceeds the observed $J$ and $H$ points. Figure $6 a$ (model 1 in Table 5) shows the model result with the addition of a circumbinary dust disk. As with EF Eri, we can easily reproduce the $8 \mu \mathrm{m}$ point, but the model underestimates the 3.6-5.8 $\mu \mathrm{m}$ points. Using a cyclotron component with $m=2$ (Fig. $6 b$ and model 2 in Table 5), the model SED has the closest match to the intermediate-wavelength data but is no longer bright enough at $8 \mu \mathrm{m}$ to match the observations. Figure $6 c$ (model 3 in Table 5) shows the $m=3$ case: larger values of $m$ cause the cyclotron peak to continue shifting toward short wavelengths until the cyclotron component appears as a

\footnotetext{
${ }^{4}$ We have used this mass in our models to predict a secondary star spectral type of M6.0 and radius of $R_{2}=0.21 R_{\odot}(\mathrm{Cox} 2000)$. However, we note that this radius is substantially larger than the calculated Roche lobe in V347 Pav assuming $M_{\mathrm{WD}}=1.00 M_{\odot}$; the secondary star radius of $R_{2}=0.16 R_{\odot}$ for this mass from Pont et al. (2005) is in better agreement with the Roche lobe size. In any case, the model SED results are not very sensitive to changes in the mass or radius of the stars.
}

Rayleigh-Jeans-like tail (similar in shape to the long-wavelength end of the secondary star SED). Figure $6 d$ (model 4 in Table 5) shows an $m=2$ cyclotron component using the single-field approach instead of the sum-over-field-strengths approach. As with EF Eri, this approach allows us to reproduce the sharp peak in the SED, which for V347 Pav is located in IRAC channel 1. However, also as with EF Eri, this approach does not reproduce the $8 \mu \mathrm{m}$ point, suggesting a combination of models 1 and 4 (i.e., dust and single-field cyclotron) as the best approach to reproducing this SED.

We now consider the possibility that the 2MASS and IRAC data were obtained at different orbital brightness states. A highstate $H$-band photometric light curve published in Bailey et al. (1995) shows that V347 Pav is variable by a factor of $\approx 4$ over its orbital period. As with EF Eri, we assume that this behavior is present in the mid-IR also and attempt to correct the 2MASS and IRAC data sets to account for orbital variability. To this end, we scaled the 2MASS flux densities by a factor of 4.0. At the nominal distance of $d=171 \mathrm{pc}$, the scaled SED can accommodate a secondary star as early as M3.5 V, whose SED exactly matches the 3.6 and $4.5 \mu \mathrm{m}$ points. However, as noted earlier in this section, the M6.0 V secondary star suggested by the mass estimate from Ramsay et al. (1996) is already at the limit of being too large for the calculated Roche lobe size, and an M3.5 V star simply will not fit into the Roche lobe of a CV with this orbital period. Consequently, we have retained the M6.0 V secondary star and adjusted the distance to the lower limit of the AraujoBetancor et al. (2005) estimate, $d=133$ pc. Figure $7 a$ shows the scaled data with a model containing a circumbinary dust disk component (model 5 in Table 5). There is considerable unmodeled flux density in the 2MASS portion of the SED, but even if we use an M3.5 V secondary star, the model SED still 
TABLE 5

Model Parameters for V347 Pay

\begin{tabular}{|c|c|c|c|c|c|c|c|}
\hline Component & Parameter $^{\mathrm{a}}$ & Model 1 & Model 2 & Model 3 & Model 4 & Model $5^{\mathrm{b}}$ & Model $6^{\mathrm{b}}$ \\
\hline System...................... & $d(\mathrm{pc})$ & 171 & 171 & 171 & 171 & 133 & 133 \\
\hline SS & Spectral type & M6.0 & M6.0 & M6.0 & M6.0 & M6.0 & M6.0 \\
\hline \multirow[t]{5}{*}{ 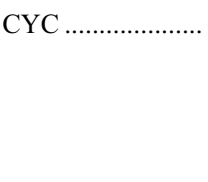 } & $B(\mathrm{MG})$ & $\ldots$ & 15,20 & 15,20 & 15,20 & $\ldots$ & 15,20 \\
\hline & $m$ & $\ldots$ & 2 & 3 & 2 & $\ldots$ & 5 \\
\hline & Sum over fields? & $\ldots$ & $\mathrm{Y}$ & $\mathrm{Y}$ & $\mathrm{N}$ & $\ldots$ & $\mathrm{Y}$ \\
\hline & $f_{\nu, \mathrm{CYC}} / f_{\nu, \mathrm{SS}}(3.6 \mu \mathrm{m})$ & $\ldots$ & 1.06 & 0.80 & 1.68 & $\ldots$ & 0.62 \\
\hline & $f_{\nu, \mathrm{CYC}} / f_{\nu, \mathrm{SS}}(8.0 \mu \mathrm{m})$ & $\ldots$ & 2.97 & 1.48 & 0.00 & $\ldots$ & 0.93 \\
\hline \multirow[t]{4}{*}{ 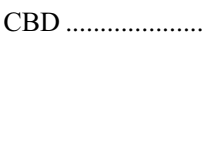 } & $r_{\text {in }}$ & $148 R_{\mathrm{WD}}$ & $\ldots$ & $\ldots$ & $\ldots$ & $148 R_{\mathrm{WD}}$ & $\ldots$ \\
\hline & $T_{\text {in }}(\mathrm{K})$ & 770 & $\ldots$ & $\ldots$ & $\ldots$ & 655 & $\ldots$ \\
\hline & $r_{\text {out }}$ & $19241 R_{\mathrm{WD}}$ & $\ldots$ & $\ldots$ & $\ldots$ & $15509 R_{\mathrm{WD}}$ & $\ldots$ \\
\hline & $T_{\text {out }}(\mathrm{K})$ & 20 & $\ldots$ & $\ldots$ & $\ldots$ & 20 & $\ldots$ \\
\hline
\end{tabular}

${ }^{\text {a }}$ Fixed model parameter values for this target include $R_{\mathrm{WD}}=0.008 R_{\odot}, R_{2}=0.21 R_{\odot}$, and $T_{2}=2850 \mathrm{~K}$. See Table 3 for the values of other parameters not listed here.

b These models refer to the scaled 2MASS data; see text.

somewhat underestimates these data points. At all values of the transition harmonic $m \geq 2$, the cyclotron component cannot match the $8 \mu \mathrm{m}$ point without exceeding the shorter wavelength IRAC points. Figure $7 b$ (model 6 in Table 5) shows a model in which the cyclotron component is partially optically thick $(m=2)$. The peak of the cyclotron emission component shifts toward longer wavelengths, leaving a deficit of modeled flux density at $8 \mu \mathrm{m}$, but suggesting a path toward recovering the previously unmodeled flux at the 2MASS wavelengths.

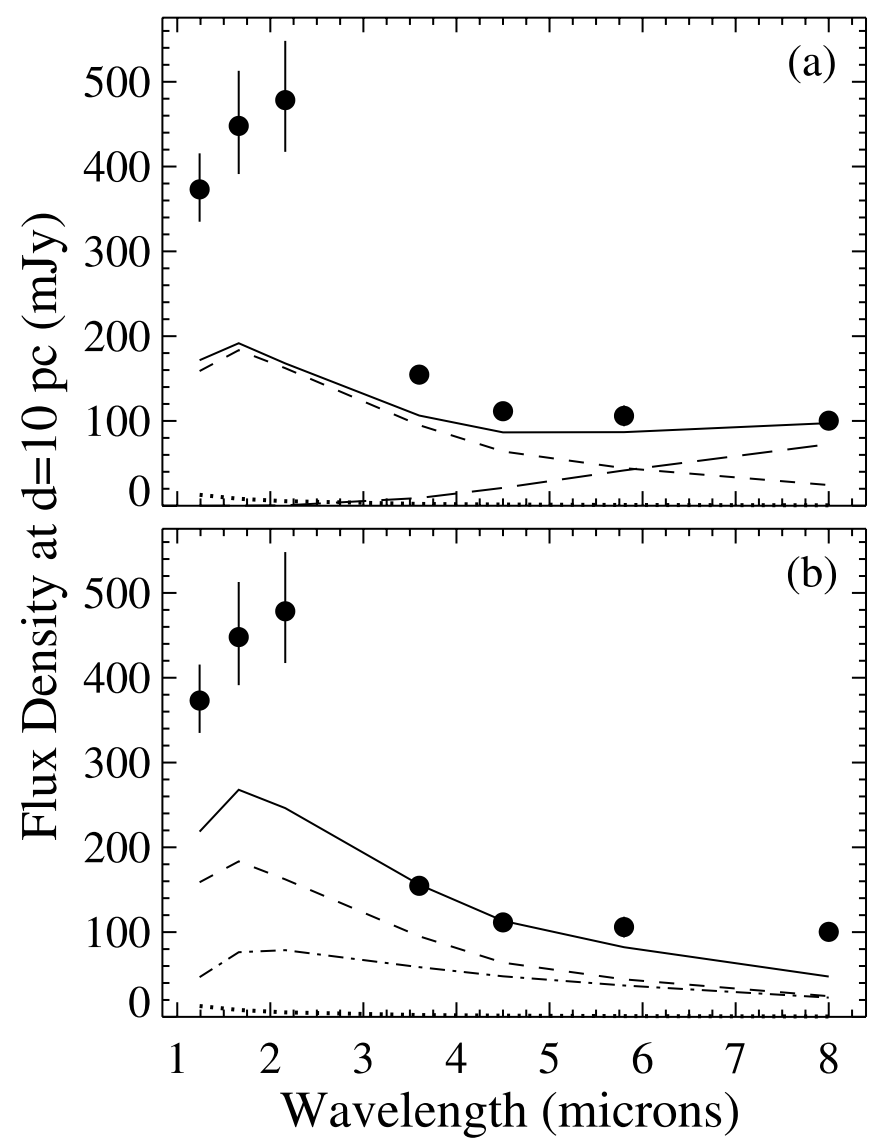

Fig. 7. - Same as in Fig. 6, but with the 2MASS data for V347 Pav scaled by a factor of 4 to account for potential orbital brightness variations. (a) Circumbinary disk model; $(b)$ cyclotron model with $m=5$. See text and Table 5 for details.

\subsection{Class II: Dominant Secondary Star}

\subsubsection{VV Puppis}

VV Pup was discovered during the early twentieth century (van Gent 1931) as a faint ( $V=14.5-18$; Downes et al. 2001), rapidly periodic $\left(P_{\text {orb }}=100.4\right.$ minutes $)$ variable star (Walker 1965). Based on photometric and spectroscopic observations, Herbig (1960) suggested that it was a binary whose emission lines originated on the brighter component. Subsequently, VV Pup was identified as the third known example of the AM Her class (Tapia 1977).

VV Pup has the distinction among our sample of polars of having the observed SED that is most reminiscent of the falling Rayleigh-Jeans tail of a cool, main-sequence star. Figure $8 a$ (model 1 in Table 6) shows a model SED that utilizes the recent tight constraints on the WD and secondary star parameters provided by Howell et al. (2006a) with a circumbinary dust disk to provide the additional flux density required to match the observed $8 \mu \mathrm{m}$ point. However, the model SED underestimates the observed flux densities from the $H$ band to $4.5 \mu \mathrm{m}$ (IRAC channel 2). This model requires a distance scaling of $d=100 \mathrm{pc}$, which is slightly more than $1 \sigma$ away from the nominal value of $d=129_{-21}^{+29} \mathrm{pc}$ from the trigonometric parallax distance reported in Howell et al. (2006a).

For all values of the transition harmonic $m \geq 2$, the peak of a cyclotron emission component shifts to successively shorter wavelengths (e.g., between the $K_{s}$ and IRAC channel 1 points for $m=2$ ), which causes the total model SED to exceed all of the shorter wavelength points when the cyclotron emission is scaled to fit the $8 \mu \mathrm{m}$ point. By moving the polar back to the nominal distance of $d=129 \mathrm{pc}$ and including a cyclotron component with $m>2$ (see, e.g., Fig. $8 b$ and model 2 in Table 6), we can mitigate this problem and produce very good model SEDs (with better agreement at 3.6 and $4.5 \mu \mathrm{m}$ and only a small flux density deficit at $8 \mu \mathrm{m}$ ). However, this form of the model is not very discriminatory, as the shape of the cyclotron component SED for $m>2$ is very similar to that of the secondary star, so this model is basically equivalent to including only the stellar components and simply scaling the distance up or down until the best match to the observed data is obtained.

\subsection{Class III: Bright 2MASS, Flat IRAC}

We suspect that the polars in class III were in high accretion states during the 2MASS observations due to the shape of the 


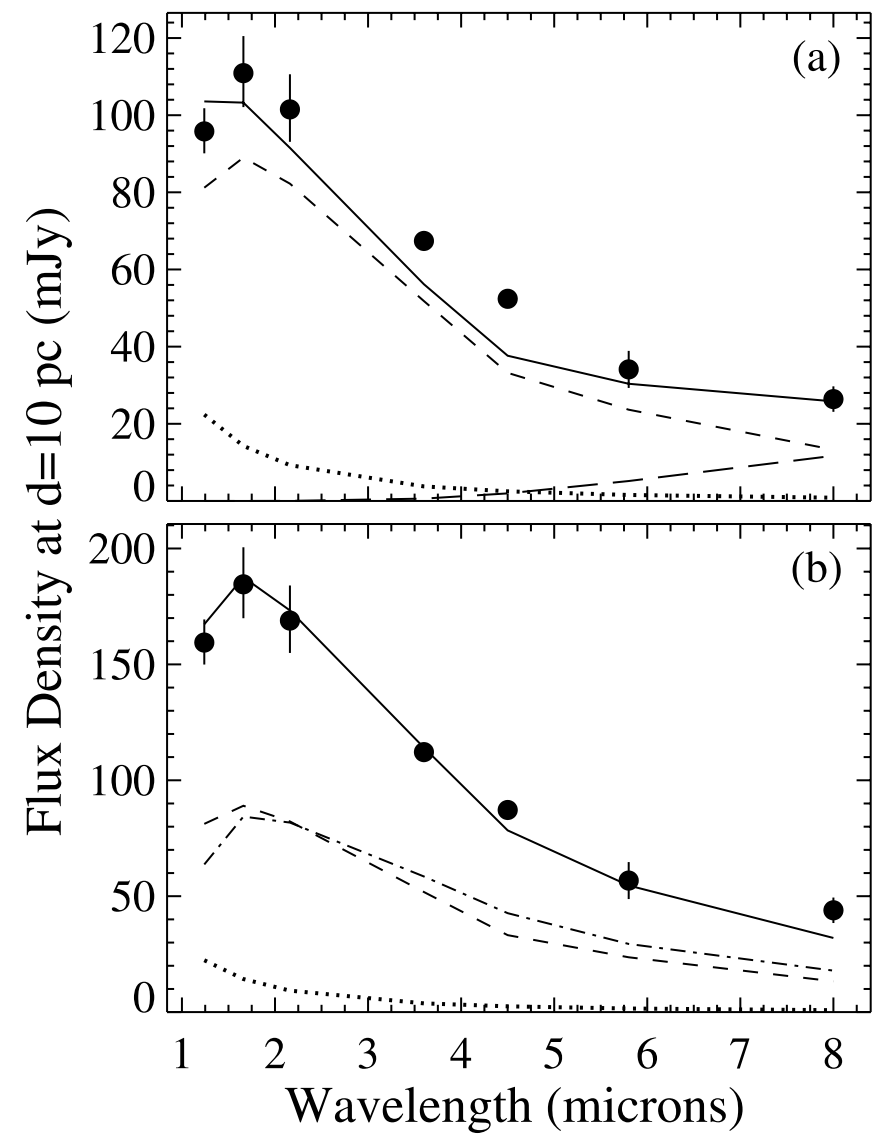

FIG. 8.- Representative model SEDs for VV Pup showing the observed data ( filled circles), total model SED (solid line), and model components: WD (dotted line), secondary star (short-dashed line), circumbinary dust disk (long-dashed line), and cyclotron emission (dot-dashed line). (a) Circumbinary disk model; (b) cyclotron model with $m=3$. See text and Table 6 for details.

bright 2MASS portion of their SEDs, which could be indicative of the falling Rayleigh-Jeans-like tail of accretion-generated luminosity. If present, then this (unmodeled) component likely contributes at 3.6 and $4.5 \mu \mathrm{m}$ in addition to dominating the 2MASS portion of the SED. We have not attempted to model this additional component because the exact characteristics of the accretiongenerated luminosity are not known in detail and would depend on a large number of (largely unknown or poorly constrained) additional parameters (e.g., mass transfer rate, plasma flow and magnetic field geometry, accretion spot size and shape). The additional complexity that would be introduced by adding another model component is not warranted for our sparsely sampled SEDs.

\subsubsection{V834 Centauri}

Mason et al. (1983) identified a 15th magnitude, blue, emissionline ( $\left.\mathrm{H}_{\mathrm{I}}, \mathrm{He}_{\mathrm{I}}, \mathrm{He} \mathrm{II}\right)$ star (subsequently named V834 Cen) as the optical counterpart of the variable X-ray source H1409-45/ E1405-451 (Jensen et al. 1982). Their time-resolved optical photometry revealed a period of $P_{\text {orb }}=101.52(2)$ minutes with an amplitude of $\sim 1 \mathrm{mag}$. In addition, rapid variability on timescales of 1-3 s was observed; this was later shown to be quasiperiodic in nature, with a coherence time of $\sim 1$ minute (Larsson 1985, 1992; Imamura et al. 2000). Wright et al. (1988) detected V834 Cen in the radio, at a peak level of $35 \mathrm{mJy}$ at $8.4 \mathrm{GHz}$. The radio flux showed variability on timescales as short as 1 minute. Based on these characteristics (coupled with its X-ray emission and circular and linear polarization observed by Tapia [1982]
TABLE 6

Model Parameters for VV Pup

\begin{tabular}{|c|c|c|c|}
\hline Component & Parameter $^{\mathrm{a}}$ & Model 1 & Model 2 \\
\hline System .................... & $d(\mathrm{pc})$ & 100 & 129 \\
\hline SS & Spectral type & M7.0 & M7.0 \\
\hline \multirow[t]{5}{*}{ CYC ....................... } & $B(\mathrm{MG})$ & $\ldots$ & 32 \\
\hline & $m$ & $\ldots$ & 3 \\
\hline & Sum over fields? & $\ldots$ & $\mathrm{Y}$ \\
\hline & $f_{\nu, \mathrm{CYC}} / f_{\nu, \mathrm{SS}}(3.6 \mu \mathrm{m})$ & $\ldots$ & 1.13 \\
\hline & $f_{\nu, \mathrm{CYC}} / f_{\nu, \mathrm{SS}}(8.0 \mu \mathrm{m})$ & $\ldots$ & 1.35 \\
\hline \multirow[t]{4}{*}{ CBD ….................. } & $r_{\text {in }}$ & $108 R_{\mathrm{WD}}$ & $\ldots$ \\
\hline & $T_{\text {in }}(\mathrm{K})$ & 510 & $\ldots$ \\
\hline & $r_{\text {out }}$ & $8140 R_{\mathrm{WD}}$ & $\ldots$ \\
\hline & $T_{\text {out }}(\mathrm{K})$ & 20 & $\ldots$ \\
\hline
\end{tabular}

${ }^{\text {a }}$ Fixed model parameter values for this target include $R_{\mathrm{WD}}=0.0105 R_{\odot}$, $R_{2}=0.15 R_{\odot}$, and $T_{2}=2670 \mathrm{~K}$. See Table 3 for the values of other parameters not listed here.

and Visvanathan \& Tuohy [1983]), Mason et al. (1983) suggested that this object was an AM Her type CV.

Recently, Mennickent et al. (2004) have matched the secondary star features in near-infrared (1-2.5 $\mu \mathrm{m})$ spectra of V834 Cen with a spectral type of M8 $\pm 0.5 \mathrm{~V}$. An M8 V star is quite faint, so we have used a minimum distance of $d=121 \mathrm{pc}$ in our models (Araujo-Betancor et al. 2005). Figure $9 a$ (see model 1 in Table 7) shows the model SED with a circumbinary dust disk, which reproduces the observed $8 \mu \mathrm{m}$ point but underestimates the flux densities at shorter wavelengths. The secondary star would contribute significantly to the total model SED only at much smaller distances $(d \approx 50 \mathrm{pc})$ than estimated in the literature. Figure $9 b$ (model 2 in Table 7) shows the model with the circumbinary disk replaced by cyclotron emission with $m=2$. The cyclotron profile peak is centered around 3.6-4.5 $\mu \mathrm{m}$, and even when the cyclotron emission is scaled up so that all of the $3.6 \mu \mathrm{m}$ flux density is accounted for, it still underestimates the $8 \mu \mathrm{m}$ point. Figure $9 \mathrm{c}$ (model 3 in Table 7$)$ shows a largely optically thick $(m=6)$ cyclotron component. In this case, the cyclotron component can account for some of the flux density in the bright 2MASS portion of the SED but still cannot reproduce the $8 \mu \mathrm{m}$ point.

\subsubsection{GG Leonis}

GG Leo was discovered by the ROSAT All Sky Survey and subsequently identified as a polar by Burwitz et al. (1998). They derived the orbital period $P_{\mathrm{orb}}=79.87946$ (7) minutes from X-ray dip timings and concluded that the shape of the light curve is consistent with accretion at only one of the WD poles. The optical light curve presented in Burwitz et al. (1998) is variable by about $1 \mathrm{mag}$ over the orbital period, and their photopolarimetry confirmed the one-pole accretion model and allowed them to derive a magnetic field strength of $B \sim 23 \mathrm{MG}$ in the accretion region. A fit to the low-state spectrum and the cyclotron emission yielded WD parameters of $0.8 M_{\odot}<M_{\mathrm{WD}}<1.2 M_{\odot}$ and $T_{\mathrm{WD}}=$ $8000 \mathrm{~K}$, although the authors note that temperatures up to $T_{\mathrm{WD}}=12,000 \mathrm{~K}$ cannot be excluded. Ramsay et al. (2004) give a revised mass estimate for the WD of $M_{\mathrm{WD}}=1.13 \pm 0.03 M_{\odot}$ based on X-ray data.

Burwitz et al. (1998) estimated the secondary star parameters from the orbital period and the radial velocity profile, adopting a radius of $R_{2} \sim 0.13 R_{\odot}$, a mass of $M_{2} \sim 0.09 M_{\odot}$, and a spectral type of M5 V or later. (We have used a spectral type of M7.5 V, which corresponds to these mass and radius values, in our models.) They then used the nondetection of spectral features expected from the secondary star to put a lower limit on the distance of 


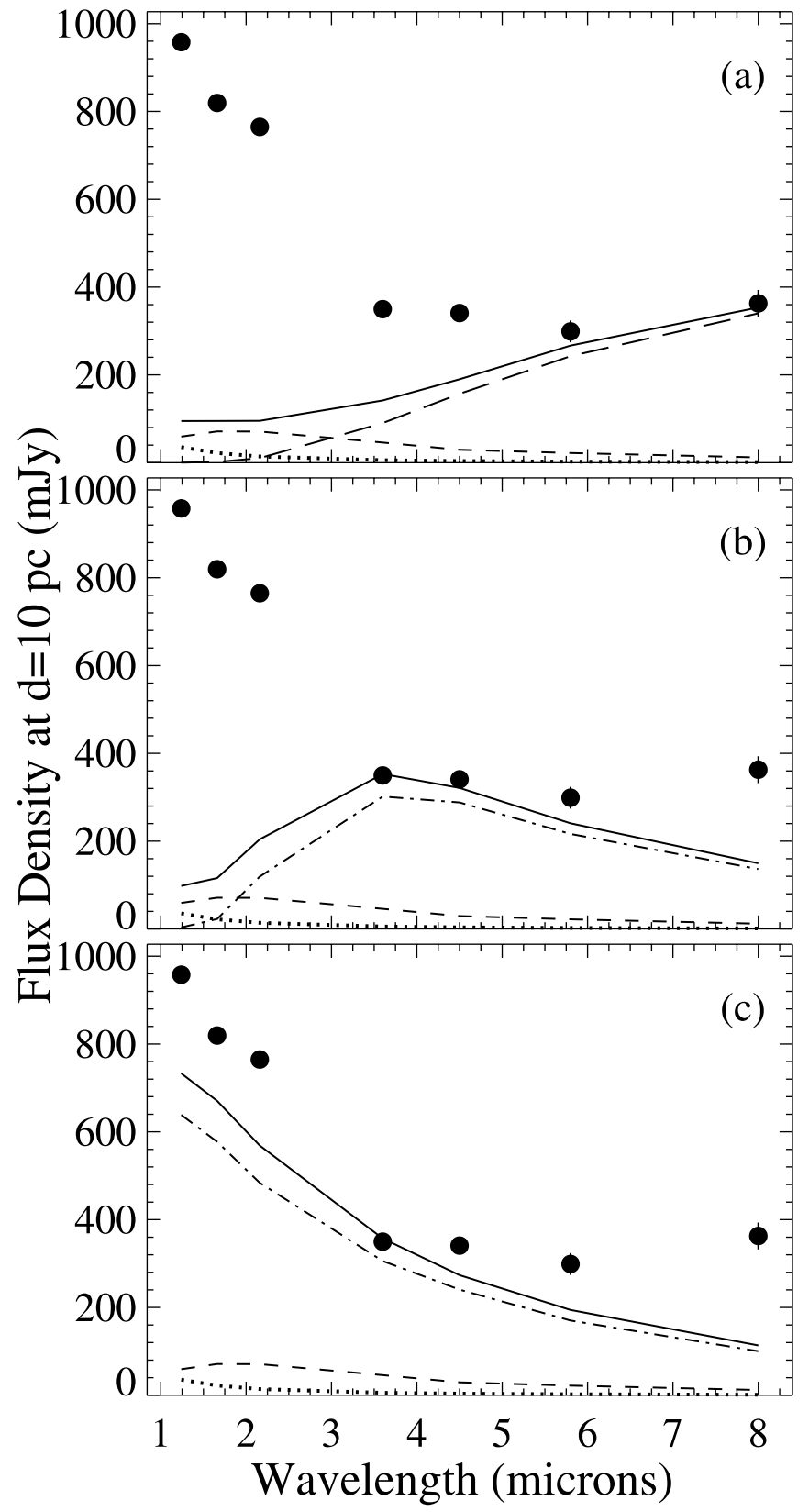

FIG. 9.- Representative model SEDs for V834 Cen showing the observed data ( filled circles), total model SED (solid line), and model components: WD (dotted line), secondary star (short-dashed line), circumbinary dust disk (longdashed line), and cyclotron emission (dot-dashed line). (a) Circumbinary disk model; $(b)$ cyclotron model with $m=2 ;(c)$ cyclotron model with $m=6$. See text and Table 7 for details.

$d=100 \mathrm{pc}$, while the upper distance limit of $d=300 \mathrm{pc}$ is based on the fact that any larger distances would require unrealistically high accretion rates. Unless the distance to GG Leo is substantially larger than $300 \mathrm{pc}$, the secondary star spectral type cannot be earlier than M4 V or the model flux at $3.6 \mu \mathrm{m}$ will exceed the observed value. In addition, no secondary star template from the Patten et al. (2006) sample (even at early spectral types) can reproduce the observed 2MASS SED without greatly exceeding the observed IRAC data. At a median distance of $d=200 \mathrm{pc}$, the M7.5 V secondary star SED underestimates the 3.6 and $4.5 \mu \mathrm{m}$ points by about $50 \%$, leaving room for the unmodeled accretion luminosity component.

As shown in Figure $10 a$ (model 1 in Table 8), a circumbinary dust disk can reproduce the $8 \mu \mathrm{m}$ point. A similar result can be
TABLE 7

Model Parameters for V834 Cen

\begin{tabular}{|c|c|c|c|c|}
\hline Component & Parameter $^{\mathrm{a}}$ & Model 1 & Model 2 & Model 3 \\
\hline System................. & $d(\mathrm{pc})$ & 121 & 121 & 121 \\
\hline 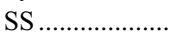 & Spectral type & M8.0 & M8.0 & M8.0 \\
\hline \multirow[t]{5}{*}{ CYC ................... } & $B(\mathrm{MG})$ & $\ldots$ & 23 & 23 \\
\hline & $m$ & $\ldots$ & 2 & 6 \\
\hline & Sum over fields? & $\ldots$ & $\mathrm{Y}$ & $\mathrm{Y}$ \\
\hline & $f_{\nu, \mathrm{CYC}} / f_{\nu, \mathrm{SS}}(3.6 \mu \mathrm{m})$ & $\ldots$ & 6.57 & 6.66 \\
\hline & $f_{\nu, \mathrm{CYC}} / f_{\nu, \mathrm{SS}}(8.0 \mu \mathrm{m})$ & $\ldots$ & 11.39 & 8.37 \\
\hline \multirow[t]{4}{*}{ 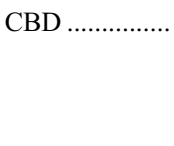 } & $r_{\text {in }}$ & $95 R_{\mathrm{WD}}$ & $\ldots$ & $\ldots$ \\
\hline & $T_{\text {in }}(\mathrm{K})$ & 875 & $\ldots$ & $\ldots$ \\
\hline & & $14663 R_{\mathrm{WD}}$ & $\ldots$ & $\ldots$ \\
\hline & $T_{\text {out }}(\mathrm{K})$ & 20 & $\ldots$ & $\ldots$ \\
\hline
\end{tabular}

${ }^{\text {a }}$ Fixed model parameter values for this target include $R_{\mathrm{WD}}=0.0115 R_{\odot}$, $R_{2}=0.10 R_{\odot}$, and $T_{2}=2490 \mathrm{~K}$. See Table 3 for the values of other parameters not listed here.

obtained even if we assume the minimum distance $(d=140 \mathrm{pc})$ at which the M7.5 V secondary star exactly matches the 3.6 and $4.5 \mu \mathrm{m}$ points. For all values of the transition harmonic $m \geq 2$, the peak of the cyclotron component is located at short wavelengths, making it impossible to reproduce the $8 \mu \mathrm{m}$ point without greatly exceeding the $3.6-5.8 \mu \mathrm{m}$ points. If we use only the single-field cyclotron prescription and assume completely optically thick emission $(m=10)$, then the resultant model trends toward reproducing the bright 2MASS portion of the SED suggesting that cyclotron emission, instead of or in addition to accretion luminosity, could also be responsible for the 2MASS data - but still cannot reproduce the $8 \mu \mathrm{m}$ point (see Fig. $10 b$ and model 2 in Table 8).

\subsubsection{MR Serpentis}

MR Ser was initially discovered in the Palomar-Green survey for UV-excess objects (Green et al. 1982); it turned out to be the only magnetic CV discovered in the Palomar-Green survey. Liebert et al. (1982) performed the first detailed observational study of MR Ser and measured an orbital period of $P_{\text {orb }}=113.56$ minutes from polarimetric observations. This was later refined by Schwope et al. (1991), who used time-resolved spectroscopic observations of a narrow emission line component (believed to originate on the secondary star) to measure $P_{\text {orb }}=113.47$ minutes (also see Schwope et al. 1993a). Wickramasinghe et al. (1991) observed shallow cyclotron humps in their optical spectra, which varied with phase in amplitude and wavelength. Harrison et al. (2005) found cyclotron emission from the $B=26 \mathrm{MG}$ WD magnetic field contaminating the blue end of their $K$-band spectrum, but the red end of the spectrum was not significantly affected by cyclotron emission.

MR Ser dropped into a low state in 1985, at which time the optical spectra showed weak $\mathrm{H} \alpha$ emission and absorption features of an M dwarf secondary star (Mukai et al. 1985). Szkody (1988) found that even in the low state, $\mathrm{H}$ and $\mathrm{He}$ emission lines were still present, indicating that there was still some ongoing accretion. Schwope et al. (1993a) identified features of the secondary star in long-wavelength optical spectra of MR Ser, corresponding to a spectral type of M5-6 V (also see Mukai \& Charles 1986). However, Harrison et al. (2005) estimated a somewhat later secondary star spectral type, M8 V, from an infrared spectrum.

Of the three polars in our class III, MR Ser and V834 Cen have the most similar observed SEDs. It is no surprise, then, that our modeling results for MR Ser are very similar to those for V834 Cen. We utilized a minimum distance of $d=134 \mathrm{pc}$ (see Table 3) in order to maximize the contribution from the faint secondary 


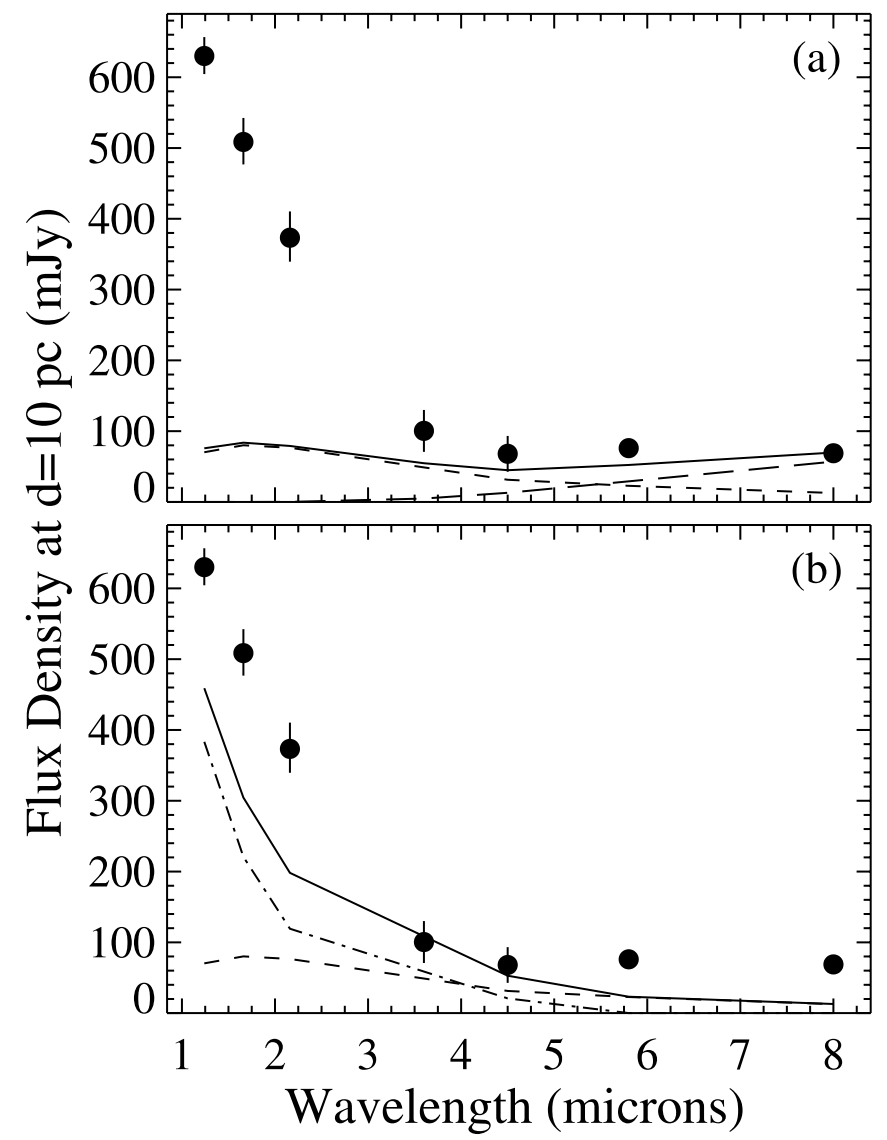

FIG. 10.-Representative model SEDs for GG Leo showing the observed data (filled circles), total model SED (solid line), and model components: secondary star (short-dashed line), circumbinary dust disk (long-dashed line), and cyclotron emission (dot-dashed line). At maximum, the WD contributes less than $1 \%$ of the total observed flux density and is not plotted. (a) Circumbinary disk model; (b) single-field cyclotron model with $m=10$. See text and Table 8 for details.

star. At this distance, the spectral type of the secondary star cannot be earlier than about M5.5 V, or the model $3.6 \mu \mathrm{m}$ point will be brighter than observed. Figure $11 a$ (model 1 in Table 9) shows the model for MR Ser containing an M7 V secondary star (consistent with the mass estimate of $M_{2}=0.1 M_{\odot}$; see Table 3) and a circumbinary dust disk. As with the similar model for V834 Cen, this reproduces the observed $8 \mu \mathrm{m}$ flux density but underestimates the flux density at all shorter wavelengths. As also found for V834

TABLE 8

Model Parameters for GG Leo

\begin{tabular}{|c|c|c|c|}
\hline Component & Parameter $^{\mathrm{a}}$ & Model 1 & Model 2 \\
\hline System................... & $d(\mathrm{pc})$ & 200 & 200 \\
\hline 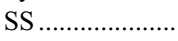 & Spectral type & M7.5 & M7.5 \\
\hline \multirow[t]{5}{*}{ CYC ...................... } & $B(\mathrm{MG})$ & $\ldots$ & 25 \\
\hline & $m$ & $\ldots$ & 10 \\
\hline & Sum over fields? & $\ldots$ & $\mathrm{N}$ \\
\hline & $f_{\nu, \mathrm{CYC}} / f_{\nu, \mathrm{SS}}(3.6 \mu \mathrm{m})$ & $\ldots$ & 1.20 \\
\hline & $f_{\nu, \mathrm{CYC}} / f_{\nu, \mathrm{SS}}(8.0 \mu \mathrm{m})$ & $\ldots$ & 0.00 \\
\hline \multirow[t]{4}{*}{ CBD $\ldots \ldots \ldots \ldots \ldots$} & $r_{\text {in }}$ & $185 R_{\mathrm{WD}}$ & $\ldots$ \\
\hline & $T_{\text {in }}(\mathrm{K})$ & 580 & $\ldots$ \\
\hline & $r_{\text {out }}$ & $16504 R_{\mathrm{WD}}$ & $\ldots$ \\
\hline & $T_{\text {out }}(\mathrm{K})$ & 20 & $\ldots$ \\
\hline
\end{tabular}

\footnotetext{
${ }^{\text {a }}$ Fixed model parameter values for this target include $R_{\mathrm{WD}}=0.006 R_{\odot}$,
} $R_{2}=0.13 R_{\odot}$, and $T_{2}=2580 \mathrm{~K}$. See Table 3 for the values of other parameters not listed here.

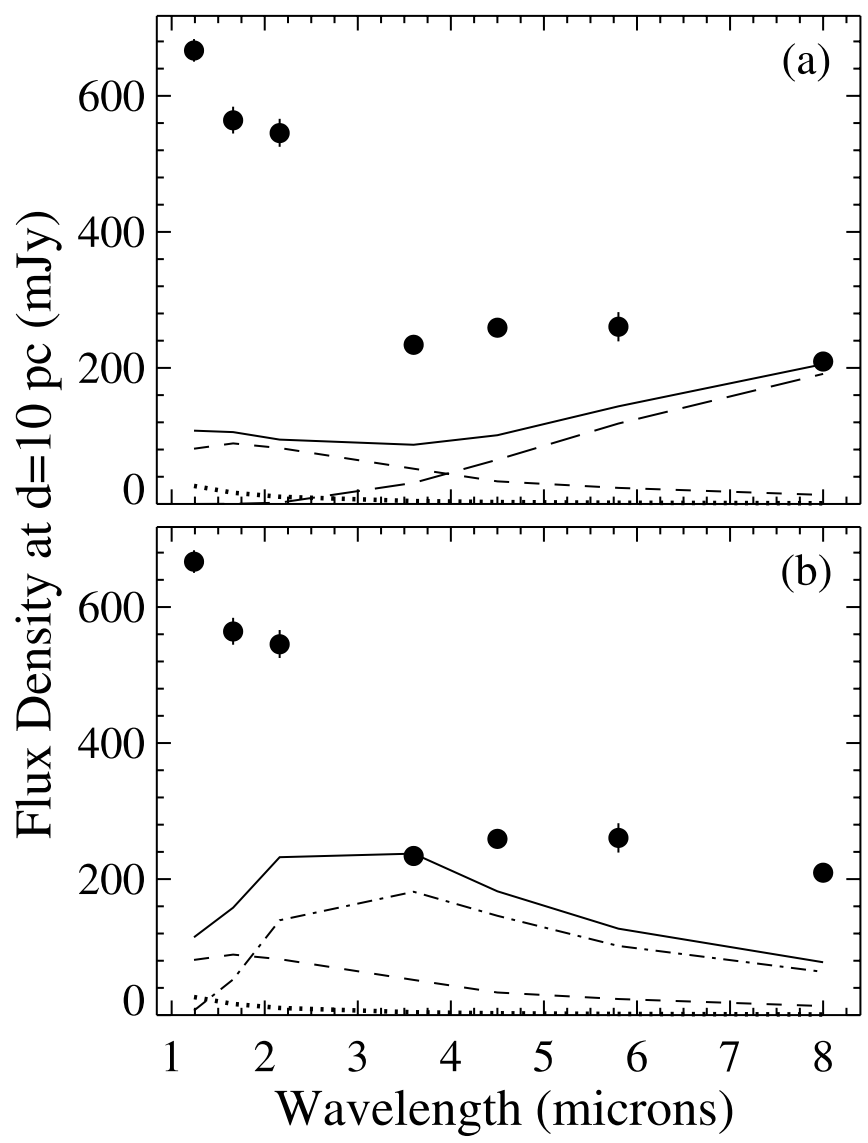

FIG. 11.- Representative model SEDs for MR Ser showing the observed data ( filled circles), total model SED (solid line), and model components: WD (dotted line), secondary star (short-dashed line), circumbinary dust disk (long-dashed line), and cyclotron emission (dot-dashed line). (a) Circumbinary disk model; (b) cyclotron model with $m=2$. See text and Table 9 for details.

Cen, at values of $m \geq 2$, the cyclotron profile peak shifts toward shorter wavelengths, making it impossible to reproduce the $8 \mu \mathrm{m}$ point without exceeding one of the shorter wavelength IRAC points (see, e.g., Fig. $11 b$ and model 2 in Table 9).

An interesting aspect of the observed IRAC SED for MR Ser is that the 4.5 and $5.8 \mu \mathrm{m}$ points are brighter than the 3.6 and $8.0 \mu \mathrm{m}$ points. This behavior is not seen in any of the other polars in our sample (see Fig. 1). Reproducing this shape is not possible using only circumbinary disk and/or cyclotron components, without invoking an additional source of flux that peaks between IRAC channels 2 and 3, for example, a blackbody component with $T \approx 800 \mathrm{~K}$ (also see the Appendix).

\subsection{Sample Best Models}

While keeping in mind our warning about the (in)ability of our data to constrain complex models, we also recognize that it is somewhat unsatisfying to just consider models that are designed to only reproduce the observed $8 \mu \mathrm{m}$ point with little regard to the SED shape at shorter wavelengths (other than to avoid exceeding the observed flux densities). To that end, we present a sample best model for each of our polars (see Table 10). These models are not constrained to be unique solutions and should not be considered as definitive physical representations of the polars. They simply illustrate that it is possible to create reasonable reproductions of the observed IR SEDs for our polars, which, given additional data, could be developed into much more physically detailed models. However, the models shown in this section do 
TABLE 9

Model Parameters for MR Ser

\begin{tabular}{clcc}
\hline \hline \multicolumn{1}{c}{ Component } & \multicolumn{1}{c}{ Parameter $^{\mathrm{a}}$} & Model 1 & Model 2 \\
\hline System ............ & $d(\mathrm{pc})$ & 134 & 134 \\
$\mathrm{SS} \ldots \ldots \ldots \ldots \ldots \ldots$ & Spectral type & $\mathrm{M} 7.0$ & $\mathrm{M} 7.0$ \\
$\mathrm{CYC} \ldots \ldots \ldots \ldots \ldots$ & $B(\mathrm{MG})$ & $\ldots$ & 28 \\
& $m$ & $\ldots$ & 2 \\
& Sum over fields? & $\ldots$ & $\mathrm{Y}$ \\
& $f_{\nu, \mathrm{CYC}} / f_{\nu, \mathrm{SS}}(3.6 \mu \mathrm{m})$ & $\ldots$ & 3.50 \\
& $f_{\nu, \mathrm{CYC}} / f_{\nu, \mathrm{SS}}(8.0 \mu \mathrm{m})$ & $\ldots$ & 4.79 \\
& $r_{\text {in }}$ & $122 R_{\mathrm{WD}}$ & $\ldots$ \\
& $T_{\text {in }}(\mathrm{K})$ & 715 & $\ldots$ \\
& $r_{\text {out }}$ & $14370 R_{\mathrm{WD}}$ & $\ldots$ \\
& $T_{\text {out }}(\mathrm{K})$ & 20 & $\ldots$ \\
\hline
\end{tabular}

${ }^{\text {a }}$ Fixed model parameter values for this target include $R_{\mathrm{WD}}=0.01 R_{\odot}$, $R_{2}=0.15 R_{\odot}$, and $T_{2}=2670 \mathrm{~K}$. See Table 3 for the values of other parameters not listed here.

not demand that the observed SEDs must be fitted using only the model components utilized here.

In all cases the best models involve combining the stellar components with cyclotron and circumbinary disk emission. Typically, the circumbinary disk emission dominates in producing the excess $8 \mu \mathrm{m}$ emission, whereas the cyclotron emission dominates at shorter wavelengths. Figure 12 shows sample best models for EF Eri (with unscaled 2MASS data), V347 Pav (unscaled), and VV Pup. In the case of EF Eri, we have optimized the model to best reproduce the IRAC SED, while neglecting the bright 2MASS $H$-band point (see $\S 4.1 .1$ for an example of fitting the $H$-band point). Figure 13 shows sample best models for V834 Cen, GG Leo, and MR Ser. In these cases, we have not attempted to reproduce the bright 2MASS portions of the SED. We can generally achieve good reproductions of the IRAC portions of the SEDs. Of course, there is likely contamination from the accretiongenerated luminosity in IRAC channels 1 and 2, which, if modeled, would cause these best models to overestimate the flux density at those wavelengths. However, this can easily be accommodated by making small adjustments to the distance, circumbinary disk inner temperature, and/or cyclotron emission strength. Out of all six of our polars, the best model for VV Pup comes closest to exactly reproducing the observed SED (see $\S 4.2$ ).

\section{DISCUSSION AND CONCLUSIONS}

We emphasize again that the results of our modeling should be considered less as specific, detailed pictures of each individual polar and more as an illustration of the general mid-IR properties of the class of polars as a whole. With that in mind, we can describe the general trends that our modeling efforts have revealed:

1. There is excess flux density at $8 \mu \mathrm{m}$ above that expected from just the stellar components in all of our target polars. Our capability to obtain sensitive IR observations has improved dramatically in recent years, especially following the launch of the Spitzer Space Telescope. Evidence for IR excess in isolated WDs is becoming increasingly common and has been convincingly linked to the presence of circumstellar dust disks. Now, CVs and LMXBs have joined the pantheon of systems containing compact objects that show excess luminosity at long wavelengths. Among the CVs, polars (which lack the bright accretion disk of nonmagnetic systems) may offer the best opportunity to detect the comparatively faint signature of circumbinary dust. The likelihood of detecting the dust emission is also improved by observing the CVs (polars or otherwise) with the shortest orbital periods (i.e., $P_{\text {orb }} \lesssim 90$ minutes), which contain the lowest mass (hence, least luminous) secondary stars.

2 . In all cases, a circumbinary dust disk can reproduce the observed $8 \mu \mathrm{m}$ flux density. Equivalent results can be obtained using either optically thick or optically thin disks. Although we have only presented model SED results from the computationally simpler optically thick disks here, we have used equivalent models containing optically thin disks to provide an estimate of the masses of dust required to explain the observed mid-IR excesses in our polars. Typical values for the total dust mass in our optically thin circumbinary disk models are $M_{\text {dust }} \sim 10^{15}-10^{17} \mathrm{~g}$, or about $10^{-19}$ to $10^{-17} M_{\odot}$. This is only a very small fraction of the annual mass transfer budget in these short orbital period $\mathrm{CVs}$, at typical rates of $\dot{M} \lesssim 10^{-11} M_{\odot} \mathrm{yr}^{-1}$ (Howell et al. 1995). It is also smaller than the $10^{20}-10^{22} \mathrm{~g}$ of dust produced during a superoutburst of a short orbital period CV (Ciardi et al. 2006). These circumbinary disks could be created (and, if necessary, replenished) from only a comparative trickle of outflowing matter over the course of millions or billions of years.

In addition, the total dust mass in our model circumbinary disks is many orders of magnitude lower than the $\sim 10^{29} \mathrm{~g}$ of material that Taam et al. (2003) require to significantly affect CV evolution. Muno \& Mauerhan (2006) also found a paucity of circumbinary material in their LMXB systems $\left(M_{\text {dust }} \sim 10^{22} \mathrm{~g}\right)$, as did Dubus et al. (2004) from their circumbinary disk model calculations for CVs $\left(M_{\text {dust }} \lesssim 10^{24} \mathrm{~g}\right)$. As noted by Muno \& Mauerhan (2006), even if the gas-to-dust mass ratio in the circumbinary disk is $100: 1$, the total disk mass is still several orders of magnitude too small to satisfy the Taam et al. (2003) condition. If our estimates

TABLE 10

Model Parameters for Best Models

\begin{tabular}{|c|c|c|c|c|c|c|c|}
\hline Component & Parameter $^{\mathrm{a}}$ & EF Eri & V347 Pav & VV Pup & V834 Cen & GG Leo & MR Ser \\
\hline 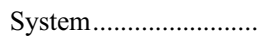 & $d(\mathrm{pc})$ & 105 & 171 & 129 & 121 & 200 & 134 \\
\hline SS & Spectral type & L5.0 & M6.0 & M7.0 & M8.0 & M7.5 & M7.0 \\
\hline \multirow[t]{5}{*}{$\mathrm{CYC}$} & $B(\mathrm{MG})$ & 13.8 & 15,20 & 32 & 23 & 25 & 28 \\
\hline & $m$ & 3 & 2 & 3 & 6 & 5 & 2 \\
\hline & Sum over fields? & $\mathrm{Y}$ & $\mathrm{N}$ & $\mathrm{Y}$ & $\mathrm{Y}$ & $\mathrm{Y}$ & $\mathrm{Y}$ \\
\hline & $f_{\nu, \mathrm{CYC}} / f_{\nu, \mathrm{SS}}(3.6 \mu \mathrm{m})$ & 3.07 & 1.20 & 1.12 & 5.23 & 0.94 & 3.11 \\
\hline & $f_{\nu, \mathrm{CYC}} / f_{\nu, \mathrm{SS}}(8.0 \mu \mathrm{m})$ & 7.06 & 0.00 & 1.33 & 6.56 & 1.19 & 4.25 \\
\hline \multirow[t]{4}{*}{ CBD } & $r_{\text {in }}$ & $73 R_{\mathrm{WD}}$ & $148 R_{\mathrm{WD}}$ & $108 R_{\mathrm{WD}}$ & $95 R_{\mathrm{WD}}$ & $185 R_{\mathrm{WD}}$ & $122 R_{\mathrm{WD}}$ \\
\hline & $T_{\text {in }}(\mathrm{K})$ & 555 & 755 & 480 & 805 & 550 & 670 \\
\hline & $r_{\text {out }}$ & $6129 R_{\mathrm{WD}}$ & $18743 R_{\mathrm{WD}}$ & $7508 R_{\mathrm{WD}}$ & $13119 R_{\mathrm{WD}}$ & $15376 R_{\mathrm{WD}}$ & $13177 R_{\mathrm{WD}}$ \\
\hline & $T_{\text {out }}(\mathrm{K})$ & 20 & 20 & 20 & 20 & 20 & 20 \\
\hline
\end{tabular}

${ }^{\text {a }}$ Parameters not listed here have the values in the corresponding table from Tables 4-9 and Table 3. 


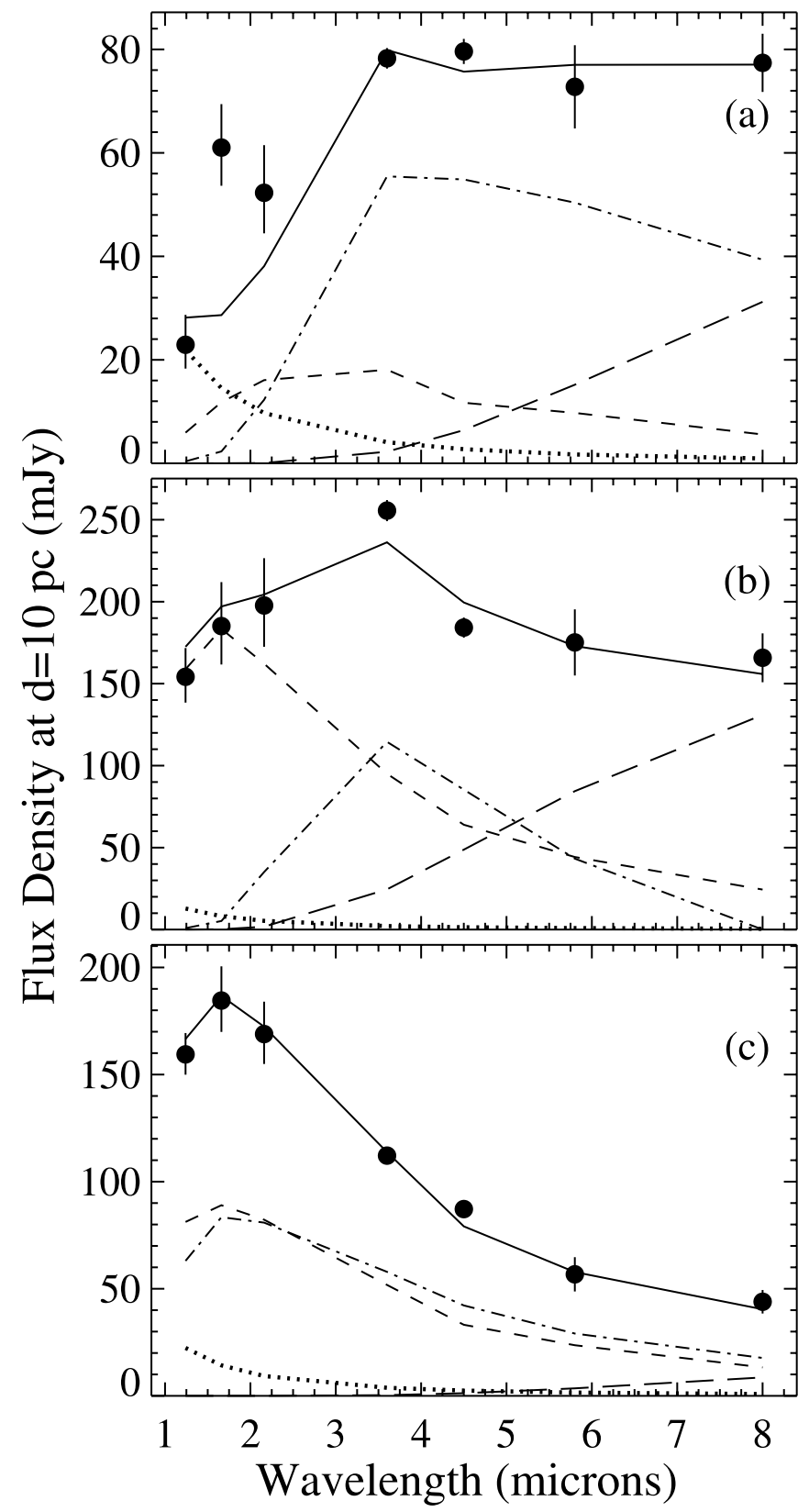

Fig. 12.- Sample model SEDs that best reproduce the observed data for (a) EF Eri (unscaled), (b) V347 Pav, and (c) VV Pup, showing the observed data ( filled circles), total model SED (solid line), and model components: WD (dotted line), secondary star (short-dashed line), circumbinary dust disk (long-dashed line), and cyclotron emission (dot-dashed line). See text and Table 10 for details.

of the circumbinary disk masses are correct, then either they cannot be responsible for the observed discrepancies in $\mathrm{CV}$ angular momentum loss rates as suggested by Taam et al. (2003) or the interaction between the circumbinary disk and the inner binary system is poorly understood.

3. On the other hand, a completely optically thin cyclotron emission model component typically has a calculated SED shape that is indistinguishable from, or very similar to, that of the circumbinary dust disk. Hence, we cannot exclude the specific case of cyclotron emission with transition harmonic $m=1$ as the source of the excess mid-IR flux density. However, when the model cyclotron emission is completely optically thin, it also does not contribute strongly at shorter wavelengths and the resultant model

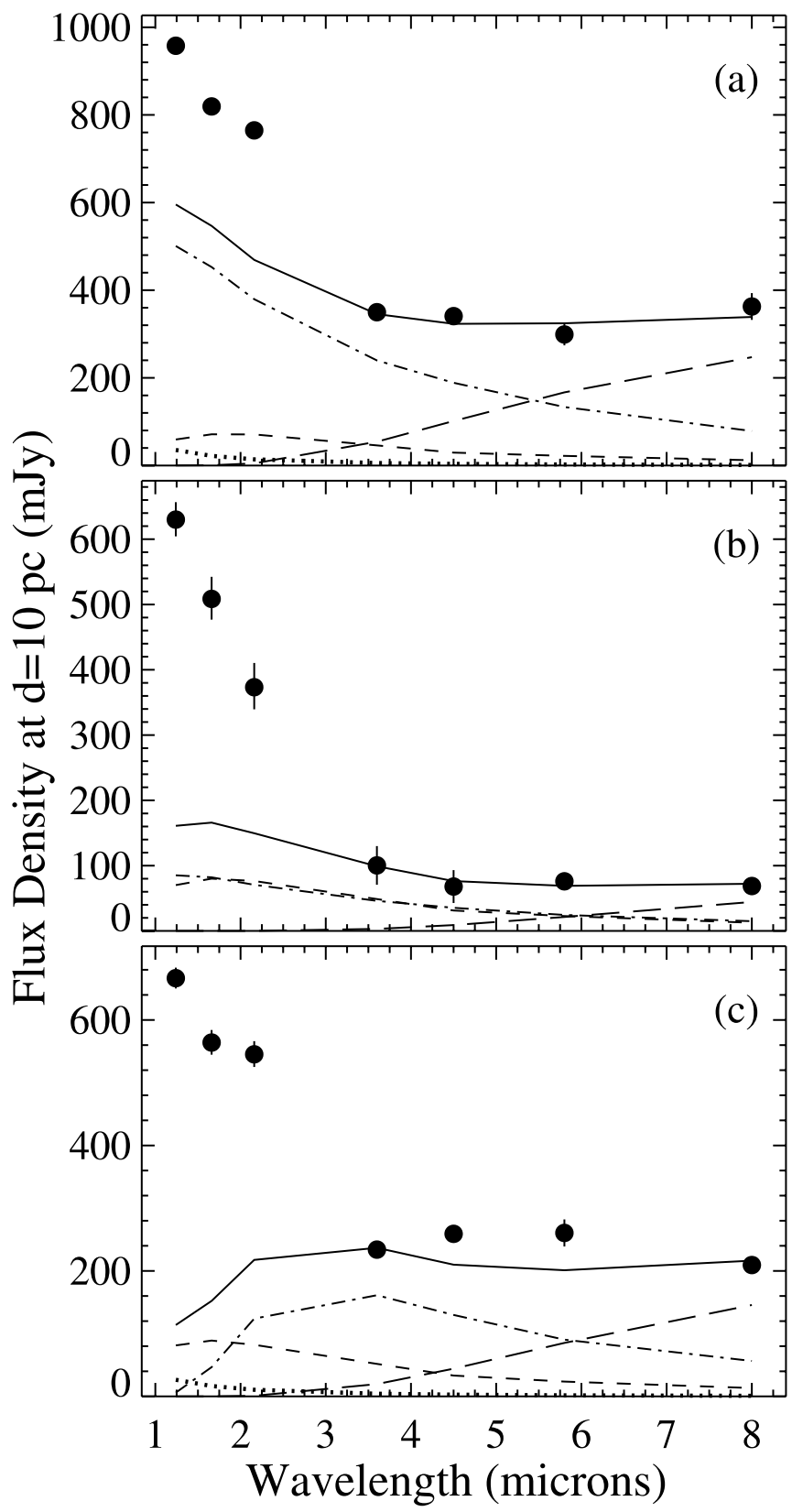

FIG. 13.- Same as in Fig. 12, but showing sample best models for (a) V834 Cen, (b) GG Leo, and (c) MR Ser. See text and Table 10 for details.

SEDs underestimate the flux density at short wavelengths. In addition, observational evidence for completely optically thin cyclotron emission has not yet been found in any polar. Since the circumbinary disk emission always contributes most strongly at $8 \mu \mathrm{m}$ (with little or no flux density contribution shortward of $3.6 \mu \mathrm{m}$ ), it seems more likely that cyclotron emission in these polars is not completely optically thin and can account for some of the shorter wavelength flux density (e.g., see the best models discussed in $\S 4.4$ ).

4. In all but one case, cyclotron emission with $m \geq 2$ is too faint at $8 \mu \mathrm{m}$ to reproduce the observed data when it is scaled so that it does not exceed any of the shorter wavelength IRAC points. The exception to this is VV Pup, which has the smallest mid-IR excess compared to the model stellar components. For this polar, optically thick cyclotron emission in combination with the stellar components can adequately reproduce the observed 
SED. However, this result for VV Pup does not really imply a preference for cyclotron emission since the optically thick cyclotron SED mimics the shape of the falling Rayleigh-Jeans tail of the secondary star SED. Hence, this model devolves to the equivalent of using only the secondary star model SED and scaling the distance to match the observed data. There are several possible reasons why VV Pup could contain the apparently smallest mid-IR excess of our sample of polars. It is probably not due to masking of the dust signature by a bright secondary star, since VV Pup does not have the longest orbital period of our sample (and, hence, does not have a correspondingly much earlier spectral type secondary star than the other polars: both MR Ser and V347 Pup have somewhat longer orbital periods and presumably similar secondary stars to VV Pup). If the IR excess is caused largely or solely by dust, then VV Pup possibly just implies that the amount or emissive characteristics of dust are not the same in all polars. The most likely explanation, however, is that VV Pup has the highest estimated inclination of our sample of polars, so the projected radiating area of the circumbinary disk is smallest.

In summary, while a circumbinary dust disk can always explain the observed $8 \mu \mathrm{m}$ excess in our target polars, only the specific case of completely optically thin cyclotron emission (which mimics the SED shape of a circumbinary disk but has not yet been observed in any polar) can also independently explain the mid-IR excess. However, even in this special case, the cyclotron emission cannot be used to account for excess flux density at shorter wavelengths (where any feasible circumbinary disk emission is also negligible). In the presence of cyclotron emission that is at all optically thick (and, hence, peaks at shorter IR wavelengths), circumbinary dust disk emission would still have to be present in order to explain the $8 \mu \mathrm{m}$ excess in our polars. If the mid-IR excess in our polars is produced, either solely or in part by dust in circumbinary disks, then the implied mass of dust is too small by many orders of magnitude to affect CV evolution as described in Taam et al. (2003).

This work is based in part on observations made with the Spitzer Space Telescope, which is operated by the Jet Propulsion Laboratory, California Institute of Technology, under a contract with NASA. Support for this work was provided by NASA through an award issued by JPL/Caltech. This publication makes use of data products from the Two Micron All Sky Survey, which is a joint project of the University of Massachusetts and the Infrared Processing and Analysis Center/Caltech, funded by NASA and the National Science Foundation. We acknowledge with thanks the variable star observations from the AAVSO International Database contributed by observers worldwide and used in this research. Last, but by no means least, we would like to thank the anonymous referee for the thoughtful and thorough review of our paper.

\section{APPENDIX}

\section{ON THE POSSIBLE PRESENCE OF A WHITE DWARF CIRCUMSTELLAR DUST DISK IN POLARS}

The SED shape produced by a single-temperature blackbody component can also be produced by an optically thick circumstellar dust disk around the WD in a polar. Such a disk is exactly analogous to the WD debris disks described in Jura (2003) and Becklin et al. (2005), which were used as the basis for our consideration of the circumbinary disks. While the presence of circumstellar disks around isolated WDs is becoming increasingly "normal" as detailed IR observations of these objects are acquired, the existence of a WD circumstellar disk in a CV would be a rather novel occurrence. Nonetheless, we briefly explore the nature and plausibility of such a system component (although further exploration of its characteristics - and even its existence-will demand more detailed mid-IR observations than are currently available).

The circumstellar disk is centered around the WD and extends from the sublimation radius close to the WD (i.e., the radius at which the disk temperature, according to eq. [1], is in the range $1000-2000 \mathrm{~K}$, typically $\sim 5 R_{\mathrm{WD}}-10 R_{\mathrm{WD}}$ ), out to the tidal truncation radius of 0.49 times the binary separation $(\approx 80 \%$ of the WD Roche lobe radius; e.g., Papaloizou \& Pringle 1977$)$. The overall hotter temperature of this circumstellar disk can produce emission at the short IRAC wavelengths with an SED shape comparable to a single-temperature blackbody component, while its smaller radiating area (compared to a circumbinary disk) does not require moving the CV to large distances in order to avoid exceeding the observed flux density levels.

It is important to clarify the nature of this circumstellar dust disk. It is more analogous to a passive planetary ring system (à la Saturn) than to a normal CV accretion disk. For example, it is assumed to be a uniform disk that is geometrically thin, with a typical thickness comparable to the size of the dust grains. Collisional interactions between dust grains are assumed to be relatively rare (compared to a viscous accretion disk). Consequently, we assume that there is no (or only negligible) accretion-generated luminosity in the circumstellar dust disk. Several potential sources of dust in a CV are listed in $\S 3.5$. We now add two more possibilities that apply particularly to dust in a circumstellar disk around the WD: (1) a tidally disrupted asteroid or comet, as described in Jura (2003) and Becklin et al. (2005), and (2) dust produced in the outer atmosphere or wind of the secondary star (Lunine et al. 1989; Allard et al. 2001; Leggett et al. 2002; Tsuji 2002) and transferred into the Roche lobe of the WD through the L1 point.

In the polars, the WD magnetic field prevents the formation of an accretion disk because the plasma emerging from the L1 point is captured onto magnetic field lines and funneled directly to the poles of the WD. This mechanism should not work efficiently to prevent the formation of a dust disk that is composed of unionized (or otherwise uncharged) material that will not interact strongly with the magnetic field. However, dust grains in space that are exposed to UV radiation will generate a flux of photoelectrons and develop a positive surface charge that rapidly reaches an equilibrium value (Horányi 1996). The amount of equilibrium charge has some dependence on the grain composition, and the grain size determines the timescale over which the equilibrium charge is reached (with larger grains reaching the equilibrium charge faster). We define the critical surface charge, $Q_{\text {crit }}$, on a dust grain such that, for larger charges, the dust will interact with the WD magnetic field in a polar and formation of a dust disk would be impeded in much the same way as for a normal CV accretion disk. For smaller charges, the dust and magnetic field will not interact appreciably, and the circumstellar dust disk can form even around the magnetic WD in a polar. 


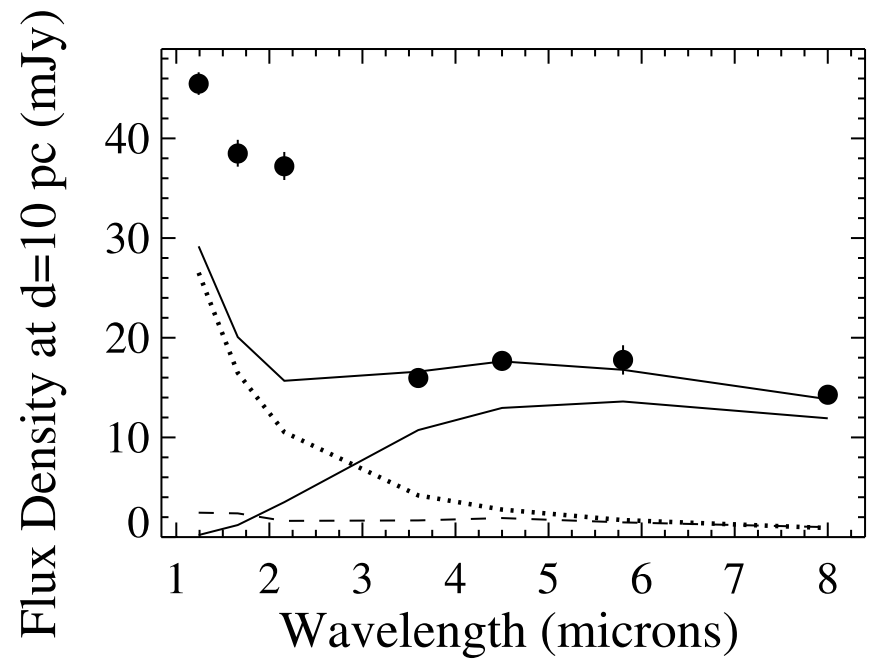

Fig. 14. - Speculative model SED for MR Ser showing the observed data ( filled circles), total model SED (solid line), and model components: WD (dotted line), secondary star (dashed line), and WD circumstellar disk (triple-dot-dashed line; see the Appendix).

By comparing the gravitational and magnetic forces acting on a dust grain emerging from L1 and undergoing free-fall acceleration toward the WD, we can estimate the value of $Q_{\text {crit }}$. The gravitational force, $F_{G}$, is given by

$$
F_{G}=\frac{G m M_{\mathrm{WD}}}{r^{2}},
$$

where $m$ is the mass of a dust grain, $M_{\mathrm{WD}}$ is the WD mass, $r$ is the distance from the center of the WD to the dust grain, and $G$ is the gravitation constant. The magnetic force acting on the dust grain, $F_{m}$, is given by

$$
F_{m}=Q v B_{\mathrm{WD}}
$$

where $Q$ is the surface charge on a grain of dust, $v$ is the free-fall velocity of the dust (itself a function of distance from the WD), and $B_{\mathrm{WD}}$ is the WD surface magnetic field (magnetic flux density). After equating these two forces, we can solve for $Q_{\text {crit }}$ to obtain

$$
Q_{\text {crit }}=\frac{\left[(1 / 2) G M_{\mathrm{WD}}\right]^{1 / 2} m R_{\mathrm{sub}}^{3 / 2}}{R_{\mathrm{WD}}^{3} B_{\mathrm{WD}}},
$$

where we have considered the maximum potential interaction between the WD magnetic field and the dust by solving for $Q_{\text {crit }}$ as close as possible to the $\mathrm{WD}$, at the dust sublimation radius, $R_{\text {sub }}$.

We have calculated values of $Q_{\text {crit }}$ using the system parameters of MR Ser and assuming $R_{\text {sub }}=7 R_{\mathrm{WD}}$, which corresponds to $T_{\text {sub }}=$ $1500 \mathrm{~K}$ (the midpoint of the possible range of sublimation temperatures). For spherical dust grains with uniform density of $3 \mathrm{~g} \mathrm{~cm}^{-3}$ and radii of 1,10 , and $100 \mu \mathrm{m}$, we obtain $Q_{\text {crit }} \approx 1.9 \times 10^{n} e$, where $n=2,5$, and 8, respectively. ${ }^{5}$ Kempf et al. (2004) measured the charge on in situ interplanetary dust grains in the solar system using the Cosmic Dust Analyzer on the Cassini spacecraft. They found values of $\sim(8-34) \times 10^{3} e$ for $\sim 10 \mu \mathrm{m}$, spheroidal grains. This is $\sim 5-25$ times smaller than our $Q_{\text {crit }}$ for similarly sized grains. Sickafoose et al. (2000) have experimentally investigated the photoelectric charging of dust grains and find an equilibrium surface charge of $\approx 4 \times 10^{4} e$ for graphite grains with radii of $\approx 50 \mu \mathrm{m}$ in the presence of $U V$ illumination with a minimum wavelength of $\approx 2000 \AA$. Our value of $Q_{\text {crit }}$ for a grain of this size is $\approx 2 \times 10^{7} e, 500$ times larger than the charge measured by Sickafoose et al. (2000). We infer that the equilibrium charge that is likely to form on dust grains in WD circumstellar disks in our polars is substantially smaller than the charge required to produce significant interaction between the dust and the WD magnetic field at distances from the WD larger than the sublimation radius. Thus, the presence of a circumstellar dust disk around the WD in a polar, while possibly improbable, is not an impossible scenario.

This calculation was motivated by the fact that, during our modeling process, we noticed that the unusual shape of the IRAC SED of MR Ser matches very closely to that expected from only a WD circumstellar disk. If the secondary star in MR Ser actually contributes very little to the total SED (or at least contributes more or less equally in all IRAC channels), then the observed mid-IR SED can be reproduced very well through the addition of only a WD circumstellar disk with an inner temperature of $1300 \mathrm{~K}$ (see Fig. 14). However, in this case, the secondary star must be exceptionally faint in order to prevent the $3.6 \mu \mathrm{m}$ flux density from becoming too bright. Even an L-type star produces too much flux at $3.6 \mu \mathrm{m}$ at the distance required to match the flux density of the WD circumstellar disk (which is

\footnotetext{
${ }^{5}$ We note in passing that our calculated values of $Q_{\text {crit }}$ are several orders of magnitude smaller than the corresponding values, $Q_{\max }$, at which the electrostatic tensile stress in the dust grain interiors would be sufficient to fracture (i.e., destroy) the grains (e.g., following the calculation in Draine \& Salpeter 1979).
} 
limited by the size of the WD Roche lobe) to the observed IRAC SED. A model with a T3.5 secondary star and a WD circumstellar disk with inner temperature of $1300 \mathrm{~K}$ produces an excellent fit to the IRAC SED, but only if the distance to the system is a mere $35 \mathrm{pc}$. This distance is a factor of $\approx 4$ smaller than the minimum distance quoted in the literature (which was calculated by Araujo-Betancor et al. [2005] from fitting the UV spectrum of the WD in MR Ser), not to mention requiring a much later spectral type for the secondary star than estimated in the literature. Consequently, we caution that, although this circumstellar disk-dominated model is suggestive, inasmuch as it reproduces the observed IRAC SED so exactly, it is overly complex for the ability of our data to constrain it and is highly speculative.

Allard, F., Hauschildt, P. H., Alexander, D. R., Tamanai, A., \& Schweitzer, A. 2001, ApJ, 556, 357

Araujo-Betancor, S., Gänsicke, B. T., Long, K. S., Beuermann, K., de Martino, D., Sion, E. M., \& Szkody, P. 2005, ApJ, 622, 589

Bailey, J. A., Ferrario, L., Wickramasinghe, D. T., Buckley, D. A. H., \& Hough, J. H. 1995, MNRAS, 272, 579

Becklin, E. E., Farihi, J., Jura, M., Song, I., Weinberger, A. J., \& Zuckerman, B. 2005, ApJ, 632, L119

Beckwith, S. V. W., Sargent, A. I., Chini, R. S., \& Güsten, R. 1990, AJ, 99, 924

Belle, K. E., Sanghi, N., Howell, S. B., Holberg, J. B., \& Williams, P. T. 2004, AJ, 128, 448

Berriman, G., Szkody, P., \& Capps, R. W. 1985, MNRAS, 217, 327

Beuermann, K., Wheatley, P., Ramsay, G., Euchner, F., \& Gänsicke, B. T. 2000, A\&A, 354, L49

Burke, J. R., \& Silk, J. 1974, ApJ, 190, 1

Burwitz, V., et al. 1998, A\&A, 331, 262

Ciardi, D. R., Wachter, S., Hoard, D. W., Howell, S. B., \& van Belle, G. T. 2006, AJ, 132, 1989

Cohen, M., Wheaton, W. A., \& Megeath, S. T. 2003, AJ, 126, 1090

Cox, A. N. 2000, Allen's Astrophysical Quantities (4th ed.; New York: AIP)

Cropper, M. 1990, Space Sci. Rev., 54, 195

Deroo, P., et al. 2006, A\&A, 450, 181

De Ruyter, S., Van Winckel, H., Dominik, C., Waters, L. B. F. M., \& Dejonghe, H. 2005, A\&A, 435, 161

De Ruyter, S., Van Winckel, H., Maas, T., Lloyd Evans, T., Waters, L. B. F. M., \& Dejonghe, H. 2006, A\&A, 448, 641

Dhillon, V. 1998, in ASP Conf. Ser. 137, Wild Stars in the Old West, ed. S. Howell, E. Kuulkers, \& C. Woodward (San Francisco: ASP), 132

Downes, R. A., Webbink, R. F., Shara, M. M., Ritter, H., Kolb, U., \& Duerbeck, H. W. 2001, PASP, 113, 764

Draine, B. T. 1989, in Infrared Spectroscopy in Astronomy, ed. B. H. Kaldeich (ESA SP-290; Noordwijk: ESA), 93

Draine, B. T., \& Salpeter, E. E. 1979, ApJ, 231, 77

Dubus, G., Campbell, R., Kern, B., Taam, R. E., \& Spruit, H. C. 2004, MNRAS, 349, 869

Fazio, G. G., et al. 2004, ApJS, 154, 10

Ferrario, L., Bailey, J., \& Wickramasinghe, D. 1996, MNRAS, 282, 218

Ferrario, L., Wickramasinghe, D. T., Bailey, J., Hough, J. H., \& Tuohy, I. R. 1992, MNRAS, 256, 252

Frank, J., King, A., \& Raine, D. J. 2002, Accretion Power in Astrophysics (Cambridge: Cambridge Univ. Press)

Gerke, J. R., \& Howell, S. B. 2006, PASP, 118, 678

Green, R. F., Ferguson, D. H., Liebert, J., \& Schmidt, M. 1982, PASP, 94, 560

Griffiths, R. E., Ward, M. J., Blades, J. C., Wilson, A. S., Chaisson, L., \& Johnston, M. D. 1979, ApJ, 232, L27

Gursky, H., et al. 1978, ApJ, 223, 973

Harrison, T. E., Howell, S. B., Huber, M. E., Osborne, H. L., Holtzman, J. A., Cash, J. L., \& Gelino, D. M. 2003, AJ, 125, 2609

Harrison, T. E., Howell, S. B., Szkody, P., \& Cordova, F. A. 2005, ApJ, 632, L123

Harrison, T. E., Howell, S. B., Szkody, P., Homeier, D., Johnson, J. J., \& Osborne, H. L. 2004, ApJ, 614, 947

Hartmann, L., et al. 2005, ApJ, 628, L147

Herbig, G. H. 1960, ApJ, 132, 76

Hessman, F. V., Gänsicke, B. T., \& Mattei, J. A. 2000, A\&A, 361, 952

Hoard, D. W., Wachter, S., Clark, L. L., \& Bowers, T. P. 2002, ApJ, 565, 511

Horányi, M. 1996, ARA\&A, 34, 383

Howell, S. B., \& Ciardi, D. R. 2001, ApJ, 550, L57

Howell, S. B., Harrison, T. E., Campbell, R. K., Cordova, F. A., \& Szkody, P. 2006a, AJ, 131, 2216

Howell, S. B., Nelson, R. A., \& Rappaport, S. 2001, ApJ, 550, 897

Howell, S. B., Rappaport, S., \& Politano, M. 1997, MNRAS, 287, 929

Howell, S. B., Szkody, P., \& Cannizzo, J. K. 1995, ApJ, 439, 337

Howell, S. B., Walter, F. M., Harrison, T. E., Huber, M. E., Becker, R. H., \& White, R. L. 2006b, ApJ, 652, 709
Imamura, J. N., Steiman-Cameron, T. Y., \& Wolff, M. T. 2000, PASP, 112, 18 Jensen, K. A., Nousek, J. A., \& Nugent, J. J. 1982, ApJ, 261, 625

Jura, M. 2003, ApJ, 584, L91

Kempf, S., et al. 2004, Icarus, 171, 317

Kirkpatrick, J. D., et al. 1999, ApJ, 519, 802

Kolb, U. 1993, A\&A, 271, 149

Kopal, Z. 1959, Close Binary Systems (London: Chapman \& Hall) . 1969, Ap\&SS, 5, 360 1970, Ap\&SS, 8, 149

Lacy, M., et al. 2005, ApJS, 161, 41

Lamb, D. Q., \& Masters, A. R. 1979, ApJ, 234, L117

Larsson, S. 1985, A\&A, 145, L1 1992, A\&A, 265, 133

Leggett, S. K., et al. 2002, ApJ, 564, 452

Liebert, J., Stockman, H. S., Williams, R. E., Tapia, S., Green, R. F., Rautenkranz, D., Ferguson, D. H., \& Szkody, P. 1982, ApJ, 256, 594

Littlefair, S. P., Dhillon, V. S., \& Martín, E. L. 2003, MNRAS, 340, 264

Lunine, J. I., Hubbard, W. B., Burrows, A., Wang, Y.-P., \& Garlow, K. 1989, ApJ, 338, 314

Makovoz, D., \& Marleau, F. R. 2005, PASP, 117, 1113

Mason, K. O., Middleditch, J., Cordova, F. A., Jensen, K. A., Reichert, G., Murdin, P. G., Clark, D., \& Bowyer, S. 1983, ApJ, 264, 575

Mennickent, R. E., Diaz, M. P., \& Tappert, C. 2004, MNRAS, 347, 1180

Mukai, K., \& Charles, P. A. 1986, MNRAS, 222, 1P

Mukai, K., Charles, P. A., \& McPherson, N. 1985, IAU Circ., 4082, 1

Muno, M. P., \& Mauerhan, J. 2006, ApJ, 648, L135

O'Donoghue, D., Mason, K. O., Chen, A., Hassall, B. J. M., \& Watson, M. G. 1993, MNRAS, 265, 545

Paczynski, B., \& Sienkiewicz, R. 1981, ApJ, 248, L27

Papaloizou, J., \& Pringle, J. E. 1977, MNRAS, 181, 441

Patten, B., et al. 2006, ApJ, 651, 502

Piirola, V., Coye, G. V., \& Reiz, A. 1987, A\&A, 186, 120

Pont, F., Bouchy, F., Melo, C., Santos, N. C., Mayor, M., Queloz, D., \& Udry, S. 2005, A\&A, 438, 1123

Potter, S. B., Cropper, M., \& Hakala, P. J. 2000, MNRAS, 315, 423

Pounds, K. A., et al. 1993, MNRAS, 260, 77

Ramsay, G., Cropper, M., Mason, K. O., Córdova, F. A., \& Priedhorsky, W. 2004, MNRAS, 347, 95

Ramsay, G., Cropper, M., Wu, K., \& Potter, S. 1996, MNRAS, 282, 726

Rappaport, S., Joss, P. C., \& Webbink, R. F. 1982, ApJ, 254, 616

Reach, W. T., et al. 2005, PASP, 117, 978

Schwope, A. D., Beuermann, K., Jordan, S., \& Thomas, H.-C. 1993a, A\&A, 278,487

Schwope, A. D., Thomas, H.-C., Beuermann, K., \& Naundorf, C. E. 1991, A\&A, 244, 373

Schwope, A. D., Thomas, H.-C., Beuermann, K., \& Reinsch, K. 1993b, A\&A, 267, 103

Sickafoose, A. A., Colwell, J. E., Horányi, M., \& Robertson, S. 2000, Phys. Rev. Lett., 84, 6034

Skrutskie, M. F., et al. 2006, AJ, 131, 1163

Smith, D. A., \& Dhillon, V. S. 1998, MNRAS, 301, 767

Spruit, H. C., \& Taam, R. E. 2001, ApJ, 548, 900

Suh, I.-S., \& Mathews, G. J. 2000, ApJ, 530, 949

Szkody, P. 1988, PASP, 100, 791

Taam, R. E., Sandquist, E. L., \& Dubus, G. 2003, ApJ, 592, 1124

Taam, R. E., \& Spruit, H. C. 2001, ApJ, 561, 329

Tapia, S. 1977, IAU Circ., 3054, 1 1982, IAU Circ., 3685, 1

Tapia, S., \& Coyne, G. V. 1980, in IAU Symp. 88, ed. M. J. Plavec, D. M. Popper, \& R. K. Ulrich (Dordrecht: Kluwer), 471

Tsuji, T. 2002, ApJ, 575, 264

van Gent, H. 1931, Bull. Astron. Inst. Netherlands, 6, 93

Van Winckel, H. 2004, Mem. Soc. Astron. Italiana, 75, 766

Visvanathan, N., \& Tuohy, I. 1983, ApJ, 275, 709 
Walker, M. F. 1965, Mitt. Konkoly Sternw. Budapest-Szabadsaghegy, 57, 1 Warner, B. 1995, Cataclysmic Variable Stars (Cambridge: Cambridge Univ. Press)

Werner, M. W., et al. 2004, ApJS, 154, 1

Wheatley, P. J., \& Ramsay, G. 1998, in ASP Conf. Ser. 137, Wild Stars in the Old West, ed. S. Howell, E. Kuulkers, \& C. Woodward (San Francisco: ASP), 446 Wickramasinghe, D. T., Cropper, M., Mason, K. O., \& Garlick, M. 1991, MNRAS, 250, 692
Wickramasinghe, D. T., \& Meggitt, S. M. A. 1982, MNRAS, 198, 975

Willems, B., Kolb, U., Sandquist, E. L., Taam, R. E., \& Dubus, G. 2005, ApJ, 635,1263

Williams, G., Johns, M., Price, C., Hiltner, A., Boley, F., Maker, S., \& Mook, D. 1979, Nature, 281, 48

Wright, A. E., Cropper, M., Stewart, R. T., Nelson, G. J., \& Slee, O. B. 1988, MNRAS, 231, 319

Young, P., \& Schneider, D. P. 1981, ApJ, 247, 960 